FSU-HEP-031231

\title{
Signals from R-parity violating top quark decays at LHC
}

\author{
A. Belyaev $\dagger$, M-H. Genest $\ddagger$, C. Leroy $\ddagger$, R. Mehdiyev $\ddagger$ * \\ $\dagger$ Physics Department, Florida State University, \\ Tallahassee, FL 32306-4350, USA. \\ ‡ Université de Montréal, Département de Physique, \\ Montréal, QC, H3C 3J7, Canada.
}

\begin{abstract}
We evaluate the potential of the CERN LHC collider to observe rare decays of the top quark in channels involving $\mathrm{R}$-parity violating $\left(R_{p}\right)$ interactions. We stress the importance of calculating top quark production and decay simultaneously as a true $2 \rightarrow 4$ process.

The process of $t \bar{t}$ pair production followed by $R_{p}$ decay of one of the top quarks is analyzed with fast detector simulation. We show that intermediate supersymmetric particles can be observed as resonances even if they are heavier than the top quark due to the significant off-shell top-quark mass effects. The approach where the top quark is produced on-mass-shell and then decays into 2- or 3-body final state would in general lead to incorrect kinematical distributions and rates. The rates of the $2 \rightarrow 4$ process with top quark production and $R_{p}$ 3-body decay depend on the total width of the heavy intermediate sfermion which could, therefore, be measured indirectly.

We find that the LHC collider offers a unique potential to study rare top quark decays in the framework of supersymmetry with broken $R$-parity for branching fractions of $\not R_{p}$ top decays as low as $\simeq 10^{-6}$.
\end{abstract}

\footnotetext{
* On leave of absence from Institute of Physics, ANAS, Baku, AZ-370143, Azerbaijan
} 


\section{Introduction}

Processes involving the top quark, the heaviest known fermion with a mass close to the scale of the electroweak symmetry breaking, offer a unique possibility to search for physics beyond the Standard Model(SM). It is well known that the SM suffers from fundamental problems such as hierarchy, fine-tuning, and absence of dark matter candidates. It is therefore believed that there must exist a more fundamental underlying theory. One of the most promising alternative models which resolves these problems is supersymmetry. The Minimal Supersymmetric extension of the Standard Model(MSSM), with the gauge group $S U(3)_{c} \times S U(2)_{L} \times U(1)_{Y}$ contains SM particles, their superpartners and an additional Higgs doublet. The MSSM possesses an additional discrete symmetry, called $R$-parity $\left(R_{p}\right)$, which conserves lepton and baryon number:

$$
R_{p}=(-1)^{3 B+L+2 S},
$$

where $B, L$ and $S$ are the baryon number, lepton number and spin, respectively. However, there is no clear theoretical preference for the status of $R_{p}$ - to be conserved or violated - in the supersymmetric theories. Therefore the answer whether the parity is realized in nature or not should be given by experiment. It is important to notice that $R_{p}$ is much less constrained in general for the 3-rd fermion-sfermion generation compared to the first two generations. Therefore the CERN LHC collider, which can be considered as a top quark factory, plays a special role as a the perfect tool for testing the conservation of $R_{p}$ in the top-stop sector. With $t \bar{t}$ production cross section of the order of $800 \mathrm{pb}$ [1], $\sim 10^{8}$ top quarks will be produced per year, assuming an integrated luminosity of $100 \mathrm{fb}^{-1}$. These high statistics will allow for precision measurements of top quark physics and in particular, for high sensitivity to rare top quark decays in a $\not R_{p}$ scenario.

Rare top-decays with $\not R_{p}$ have been intensively studied for the last decade, and even before the discovery of the top quark [2]. If sfermions are light enough, the 2-body decay of top quark into fermion-sfermion pair will take place [3, 4, 5, 6, 7, 8, ?, ?], otherwise the decay with a single neutralino $\left(\chi^{0}\right)$ (hereafter we are using $\chi^{0}$ notation for the lightest neutralino) in the final state proceeds with the kinematics of a 3-body decay [8, 9].

The aim of the present paper is to take a closer look at $\not h_{p}$ 3-body top quark decay expanding on previous studies in the following way:

- we treat top quark production and decay coherently (i.e. calculate diagrams with off-mass-shell top quark) and show the crucial role of this approach for the predictions of kinematical distributions and production rates;

- we perform a simulation of the processes at detector level, thus obtaining realistic predictions for the reach at the LHC.

The outline of the paper is as follows: In Sect. 2, we evaluate production rates for the process $p p \rightarrow t \tilde{\chi}^{0} q \ell$. We show that only by calculating together top quark 
production and decay do we obtain the correct cross section. We scan the $\mu-M_{2}$ plane and present parton-level kinematical distributions. Sect. 3 gives details of the fast Monte-Carlo simulation, including the effect of initial and final state radiation, as well as hadronization effects, followed by the fast detector simulation. In Sect. 4, we describe the analysis procedure for extracting the signal from backgrounds and reconstructing the top quark and the slepton. In Sect. 5, we estimate the sensitivity of the LHC for observing of $\not R_{p}$ top quark decays and we draw our conclusions in Sect. 6 .

\section{Top quark production and decay: $h_{p}$ scenario}

The $R_{p}$ superpotential of the MSSM can be written as [10, 11, 12]

$$
\mathcal{W}_{\not R_{p}}=\lambda_{i j k} L_{i} L_{j} \bar{E}_{k}+\lambda_{i j k}^{\prime} L_{i} Q_{j} \bar{D}_{k}+\lambda_{i j k}^{\prime \prime} U_{i}^{c} D_{j}^{c} \bar{D}_{k}+\mu_{i} L_{i} H_{2},
$$

where $L_{i}\left(Q_{i}\right)$ and $E_{i}\left(U_{i}, D_{i}\right)$ are the left-handed lepton (quark) doublet and right-handed lepton (quark) singlet chiral superfields; $H_{2}$ is the second Higgs doublet field; $\mu_{i}$ are bilinear $R_{p}$ couplings; $i, j, k$ are generation indices. Terms with $\lambda$ and $\lambda^{\prime}$ coefficients violate lepton number while the term with coefficient $\lambda^{\prime \prime}$ violates baryon number. Lepton- and baryon- number violating operators together would lead to fast proton decay. It has been shown [13, 14, 15] that there exist symmetries that allow the $\not R_{p}$ for the subset of these operators consistent with the limits on proton decay. Therefore, here we assume hereafter that the baryon-number violating operator is vanishing. The alternative case, with non-vanishing baryon-number violating operator but no lepton-number violating operator, leads to hadronic top quark decay, which is very difficult to observe due to the huge QCD background.

The last bi-linear $R$-parity violating (BRPV) term in the superpotential (2) is responsible for spontaneous $R_{p}$ violation. In general, it cannot be rotated away from the superpotential together with the SUSY soft breaking term by a suitable choice of the basis [16]. If one tried to do this, then $R_{p}$ terms would be reintroduced via two trilinear terms given by $L L \bar{E}$ and $L Q \bar{D}$ in equation (2). Since our specific study of $\not_{p}$ involves only the top quark decay, the $L Q \bar{D} \not R_{p}$ operator (precisely, $L Q_{3} \bar{D}$ ) is given by the second term of (2), which also can effectively arise from BRPV term. It leads to the following Lagrangian in four-component Dirac notation:

$$
\begin{aligned}
L_{\not R p}= & \lambda_{i j k}^{\prime}\left[\tilde{\nu}_{i L} \bar{d}_{k R} d_{j L}+\tilde{d}_{j L} \bar{d}_{k R} \nu_{i L}+\tilde{d}_{k L}^{*} \overline{\left(\nu_{i L}\right)^{C}} d_{j L}-\right. \\
& \left.\tilde{e}_{i L} \bar{d}_{k R} u_{j L}-\tilde{u}_{j L} \bar{d}_{k R} e_{i L}-\tilde{d}_{k L}^{*} \overline{\left(e_{i L}\right)^{C}} u_{j L}\right]+ \text { h.c. }
\end{aligned}
$$

Since we study $R_{p}$ in the third family, only $\lambda_{i 3 k}^{\prime}$ couplings are relevant. They give rise to top quark decay into a single neutralino via the Feynman diagrams shown in Fig. [1. Three kinds of virtual fermions can be present in the diagrams for non-vanishing $\lambda_{i 3 k}^{\prime}$ : a slepton, a squark of the first or second generation and a stop-quark.

The decay width for this process of top-quark decay depends on: 

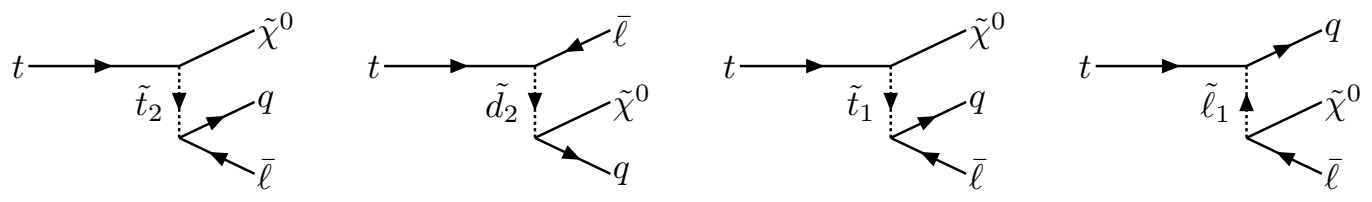

Figure 1. Tree-level diagrams for three-body top quark decay into neutralino $\left(\tilde{\chi}^{0}\right)$, lepton (l) and quark (q) via $R_{p}$ operators.

- The magnitudes of the neutralino and chargino masses and couplings, which are functions of the MSSM parameters. Assuming gaugino-mass unification, the $U(1)$ and $S U(2)$ gaugino masses $M_{1}$ and $M_{2}$ are related by $M_{1}=\frac{5}{3} \tan \theta_{W}^{2} M_{2}$, in which case the gaugino and higgsino masses and couplings are determined by $M_{2}-S U(2)$ gaugino mass, $\mu$-superpotential Higgs mass parameter and $\tan \beta$.

- The masses of the intermediate sfermion - squarks and sleptons - which could be either lighter or heavier than the top-quark.

We assume here for simplicity the case where one sfermion, for example, a slepton, is lighter than any other intermediate sfermions, i.e. it gives the leading contribution to the $\not R_{p}$ decay of the top quark. For our calculations we have used CompHEP v33.23 [17] together with a fully implemented model of $R_{p}$ (Eq 2) interactions [18]. Contours of the partial width, $\Gamma_{\text {top }}$, for top decay by the channel $t \rightarrow \tilde{\chi}^{0} \ell q$ in the $\left(\mu-M_{2}\right)$ plane are shown in Fig. 2. The partial width was calculated as in 9 .
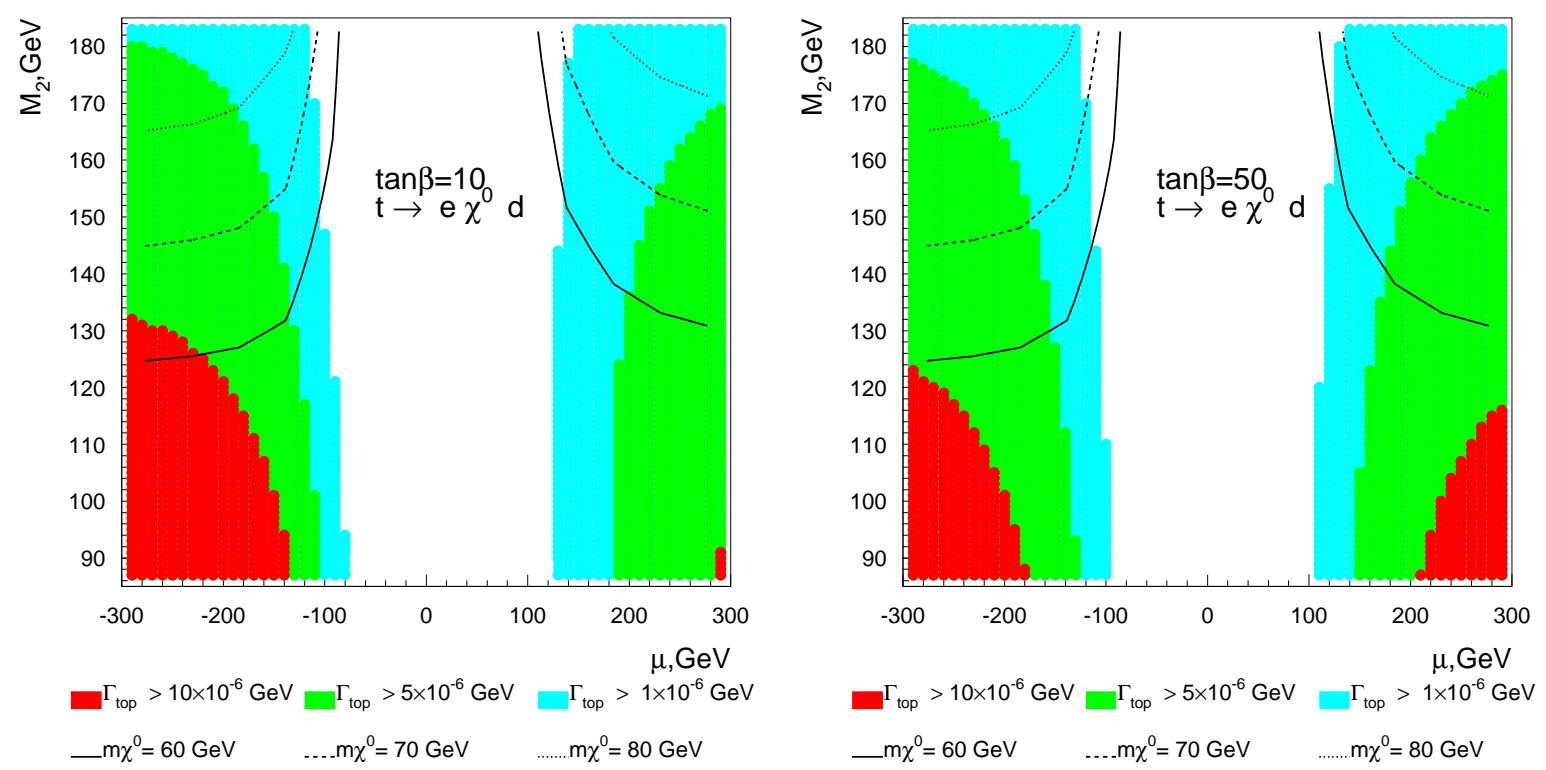

Figure 2. Contours for the partial 3-body top decay width $\Gamma_{t o p}\left[t \rightarrow \tilde{\chi}^{0} \ell q\right]$ for a slepton mass $m_{\tilde{l}}=200 \mathrm{GeV}$

The decay width decreases as $M_{2}$ increases since the neutralino mass increases with this parameter. The cross section of the complete $2 \rightarrow 4$ process $g g \rightarrow t \tilde{\chi}^{0} \ell q$ 
describing $t \bar{t}$ production and $\not R_{p}$ decay of one of top quarks can now be evaluated. The corresponding diagrams are shown in Fig. 3.
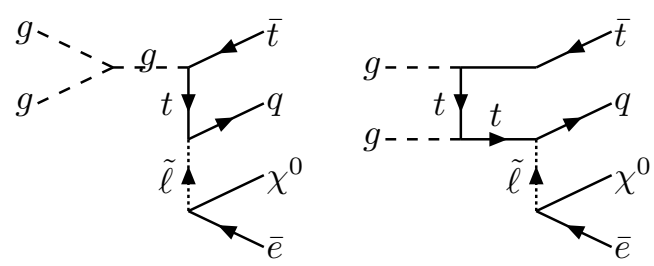

diagr.1

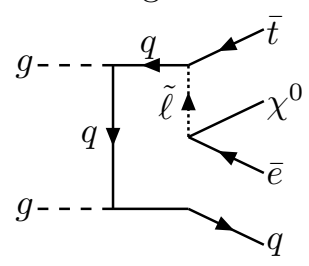

diagr.5

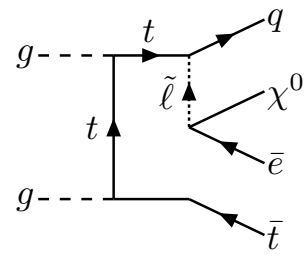

diagr.2

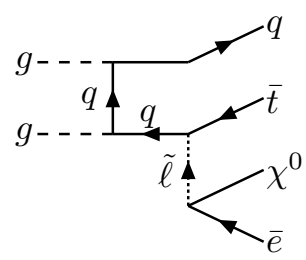

diagr.6 diagr.3

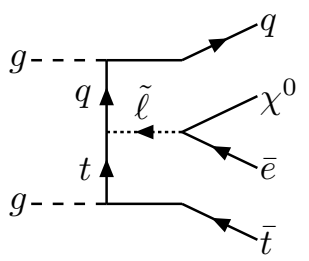

diagr.7

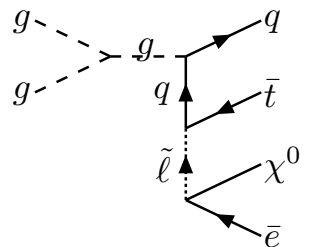

diagr.4

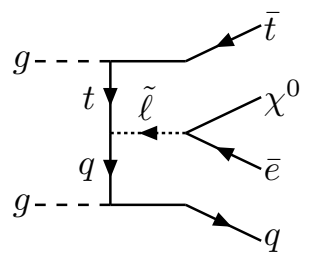

$\operatorname{diagr.8}$

Figure 3. Tree-level diagrams for the $g g \rightarrow t \tilde{\chi}^{0} \ell q$ process of $t \bar{t}$ production and decay due to $R_{p}$ interactions

For the parton-level calculations, we used the CTEQ5L [19] parton density function with the top quark mass as the QCD scale. In order to avoid soft and collinear divergences when massless quarks are present in the final state, we applied a quark transverse momentum cut, $p_{T}^{q}>10 \mathrm{GeV}$. Diagrams (1-3) in Fig. 3 form a gauge-invariant subset which does not require the $p_{T}^{q}$ cut. However, the total set of subprocesses leading to $t \tilde{\chi}^{0} \ell q$ state consists of 8 Feynman diagrams which should be calculated together.

In Fig. 4, we plot the cross section for the process $g g \rightarrow t \tilde{\chi}^{0} \ell q$ as a function of the slepton mass under three approaches:

(i) $g g \rightarrow t \bar{t} \times \operatorname{Br}[t \rightarrow q \tilde{\ell}] \times \operatorname{Br}\left[\tilde{\ell} \rightarrow \ell \chi^{0}\right]\left(m_{\tilde{\ell}}<m_{t o p}\right)$ or $g g \rightarrow t \bar{t} \times \operatorname{Br}\left[t \rightarrow q \ell \chi^{0}\right]$ $\left(m_{\tilde{\ell}}>m_{t o p}\right)$. Here, the top quark undergoing $R_{p}$ decay stays on-shell

(ii) contribution of diagrams (1-3) only

(iii) complete calculation of the process $g g \rightarrow t \tilde{\chi}^{0} \ell q$, i.e. calculations of the set of diagrams (1-8).

One can see that the cross section evaluated in approach (i) agrees within 30-50\% with those of (ii) or (iii) when $m_{\tilde{\ell}}<m_{t o p}$. On the other hand, when $m_{\tilde{\ell}}>m_{t o p}$ approach (i) underestimates the true cross section of approach (iii) by about an order of magnitude! Rates predicted by approach (ii) are about a factor of 2.5 smaller compared with those of approach (iii). To understand the origin of the difference between approaches (i) and (iii), we studied kinematical distributions. Fig. 5 shows the $\tilde{\chi}^{0} \ell$ (top) and $\tilde{\chi}^{0} \ell q$ (bottom) invariant mass distributions for the complete calculation of (iii).

Even for a quite heavy slepton $\left(m_{\tilde{\ell}}=200 \mathrm{GeV}\right)$, the resonant peak from the slepton in $\tilde{\chi}^{0} \ell$ distribution is clearly seen. This means that the slepton forces the top 


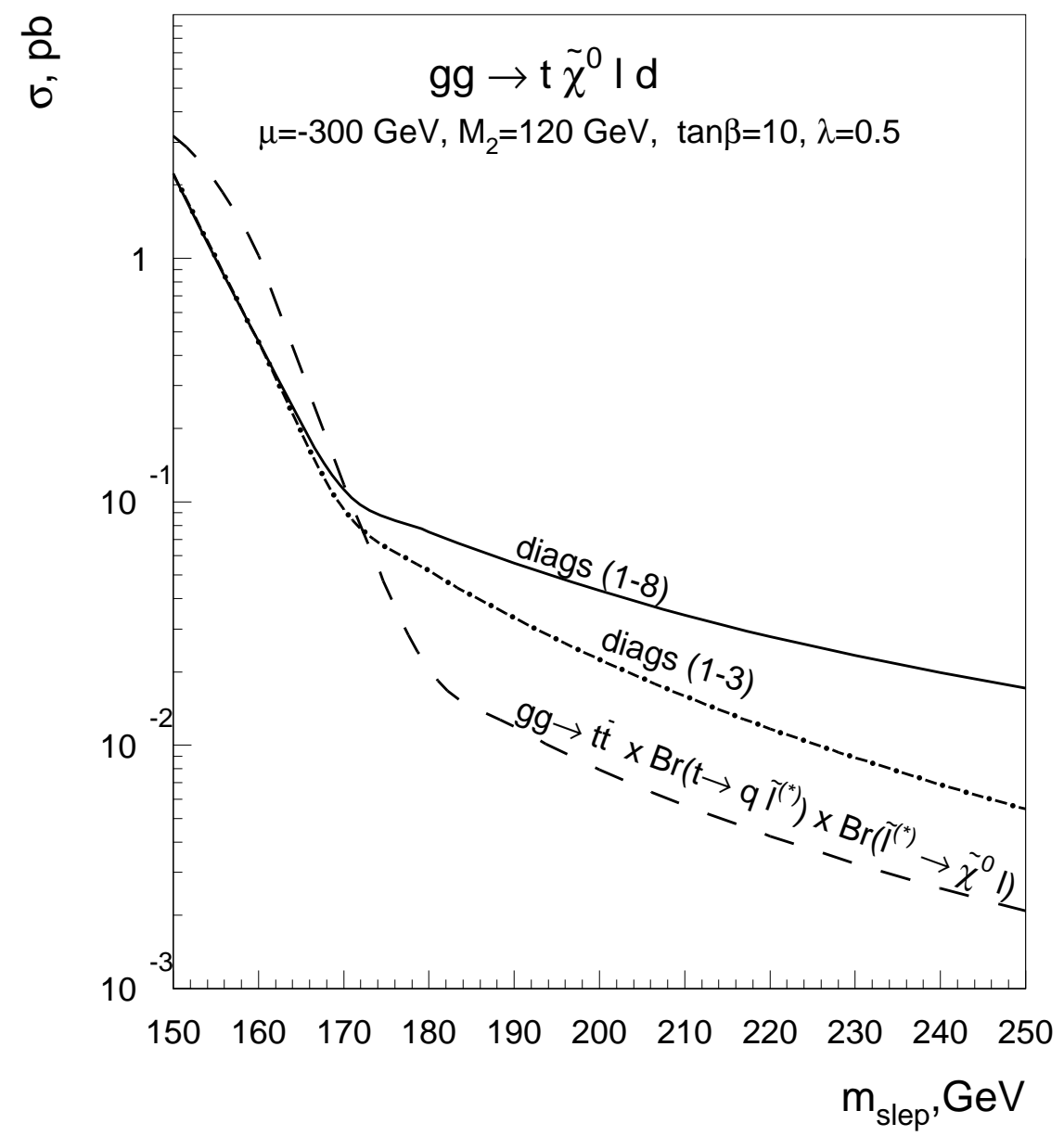

Figure 4. Cross section of $g g \rightarrow t \tilde{\chi}^{0} \ell q$ process as a function of the slepton mass for the 3 approaches (see text).

quark to be off-shell and becomes the resonance itself. The contribution from the onshell heavy slepton is significant. In approach ii.) the $\tilde{\chi}^{0} \ell$ distribution is similar, but the slepton resonance peak is slightly suppressed.

The $q \tilde{\chi}^{0} \ell$ invariant mass distribution (bottom of Fig. [5) in the top quark decay is sensitive to the mass of the intermediate slepton. For a heavy slepton $m_{\tilde{\ell}} \geq m_{\text {top }}$, the top quark resonance peak is strongly suppressed and a right shoulder appears in the $\ell \tilde{\chi}^{0} q$ invariant mass spectrum. This happens because the heavy slepton "pushes" the top quark to be off-shell and becomes itself on-shell. In this case, the experimental reconstruction of the top quark mass is more difficult due to this shoulder. On the contrary, if the slepton mass is less than the top quark mass, two-body top quark decay takes place and one can observe a pronounced peak in the $\ell \tilde{\chi}^{0} q$ invariant mass distribution.

We have explicitly checked that total cross sections for approaches (i), (ii) and (iii) agree fairly well (at about $20 \%$ level) after the application of a $20 \mathrm{GeV}$ mass window 

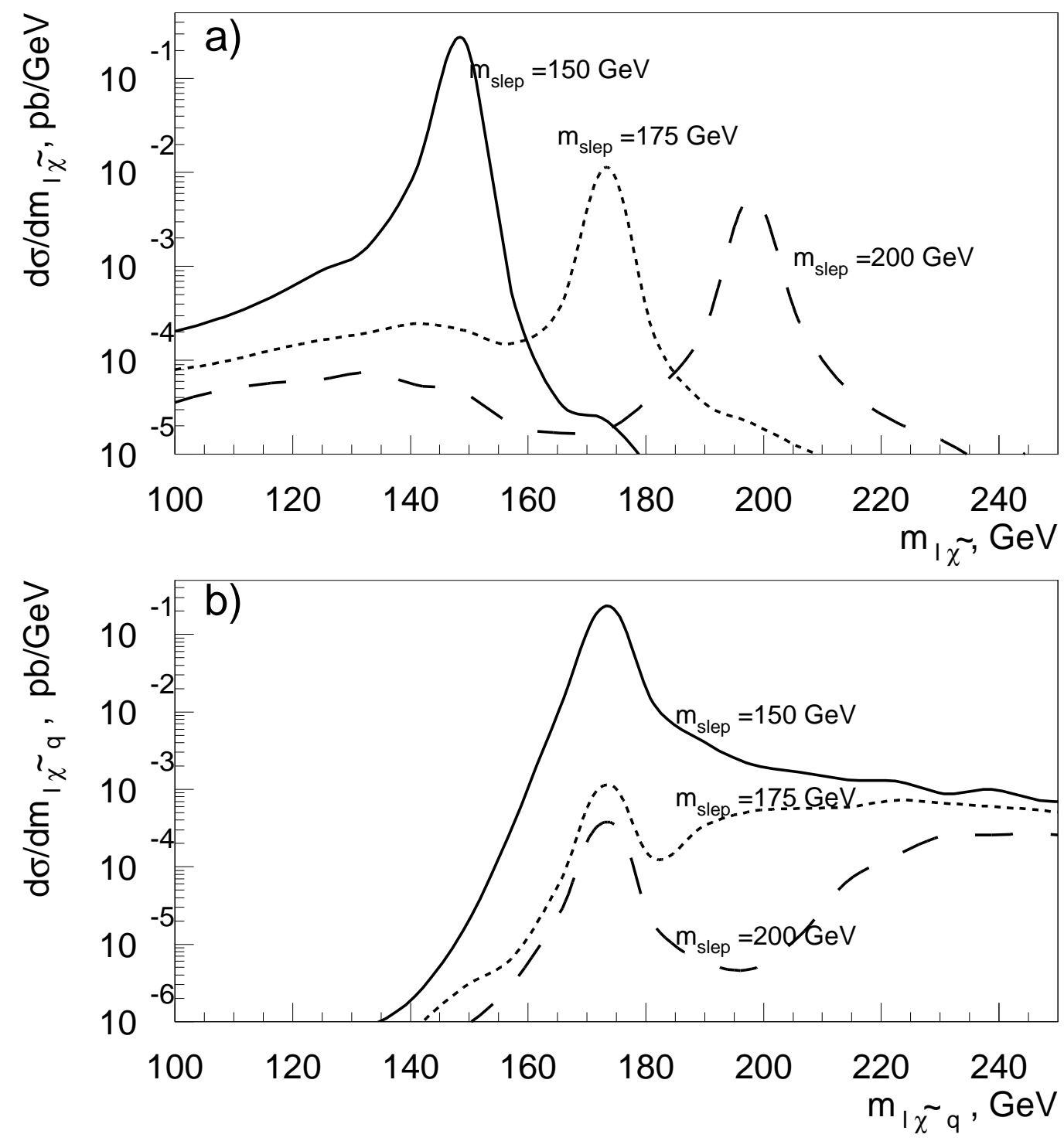

Figure 5. Parton-level $\tilde{\chi}^{0} \ell$ invariant mass distribution (top) and $\tilde{\chi}^{0} \ell q$ invariant mass distribution(bottom) for different slepton masses.

cut on $\ell \tilde{\chi}^{0}$ invariant mass to remove the contribution from the slepton.

Another difference between the predictions of approaches (i) and (iii) must be pointed out. In Fig. 2, one can see that the $R_{p}$ top decay width decreases with an increase of the $M_{2}$ parameter. Therefore, in approach (i), the cross section for the $g g \rightarrow t \tilde{\chi}^{0} \ell q$ process also decreases as $M_{2}$ increases. The situation is just the opposite in approach (iii), because the cross section of the true $2 \rightarrow 3$ process also depends on the slepton width. In Fig. 6, one can see that the cross section increases with $M_{2}$ due a decrease of the slepton width. 
For the rest of the analysis, we use approach (iii) which gives the correct cross section and kinematical distributions.

One should also note that we treated neutralino as an on-shell particle. It decays through the intermediate sparticle, which could also, in principle, "push" a neutralino to be off-shell and invalidate the on-shell approach for the neutralino. However one should recall that neutralino width is at least several orders of magnitude smaller than the width of the intermediate scalar $\left(\sim 10^{-6}-10^{-8} \mathrm{GeV}\right.$ for $\lambda \sim 0.1-0.2$ and $m_{\tilde{q}, \tilde{l}} \sim 100-200$ $\mathrm{GeV}) . \sharp$ This fact eliminates the probability for the intermediate sparticle to be on-shell for the $\tilde{\chi}^{0} \rightarrow \ell^{+}\left(\tilde{\ell_{*}^{-}}\right) \rightarrow d q$ chain. The asteriks here denotes an off-shell intermediate sparticle. We have checked this qualitative argument numerically and have calculated the width and mass distributions for the $t \rightarrow d\left(\tilde{\ell^{+}}\right) \rightarrow \ell^{+}\left(\chi^{0}\right) \rightarrow \ell^{+}\left(\tilde{\ell_{*}^{-}}\right) \rightarrow d q$ process for true $1 \rightarrow 5$ top quark decay. We have chosen $m_{\tilde{l}}=150 \mathrm{GeV}$ and have found that, indeed, the invariant mass distribution of the $l d q$ system from $\tilde{\chi}^{0} \rightarrow \ell^{+}\left(\tilde{\ell_{*}^{-}}\right) \rightarrow d q$ process, has a delta-function-like shape and the exact width for the $1 \rightarrow 5$ top quark decay can be accurately (within numerical errors of $1 \%$ ) reproduced by product of the $1 \rightarrow 3$ top quark decay width and the neutralino branching ratio. This validates the treating of neutralino as on-shell particle and suffices dealing with 3-body top decay.

In the next section, we perform a fast Monte Carlo simulation of the $g g \rightarrow t \tilde{\chi}^{0} \ell q$ process in order to estimate a realistic experimental sensitivity to $\mathbb{R}_{p}$ top quark decay.
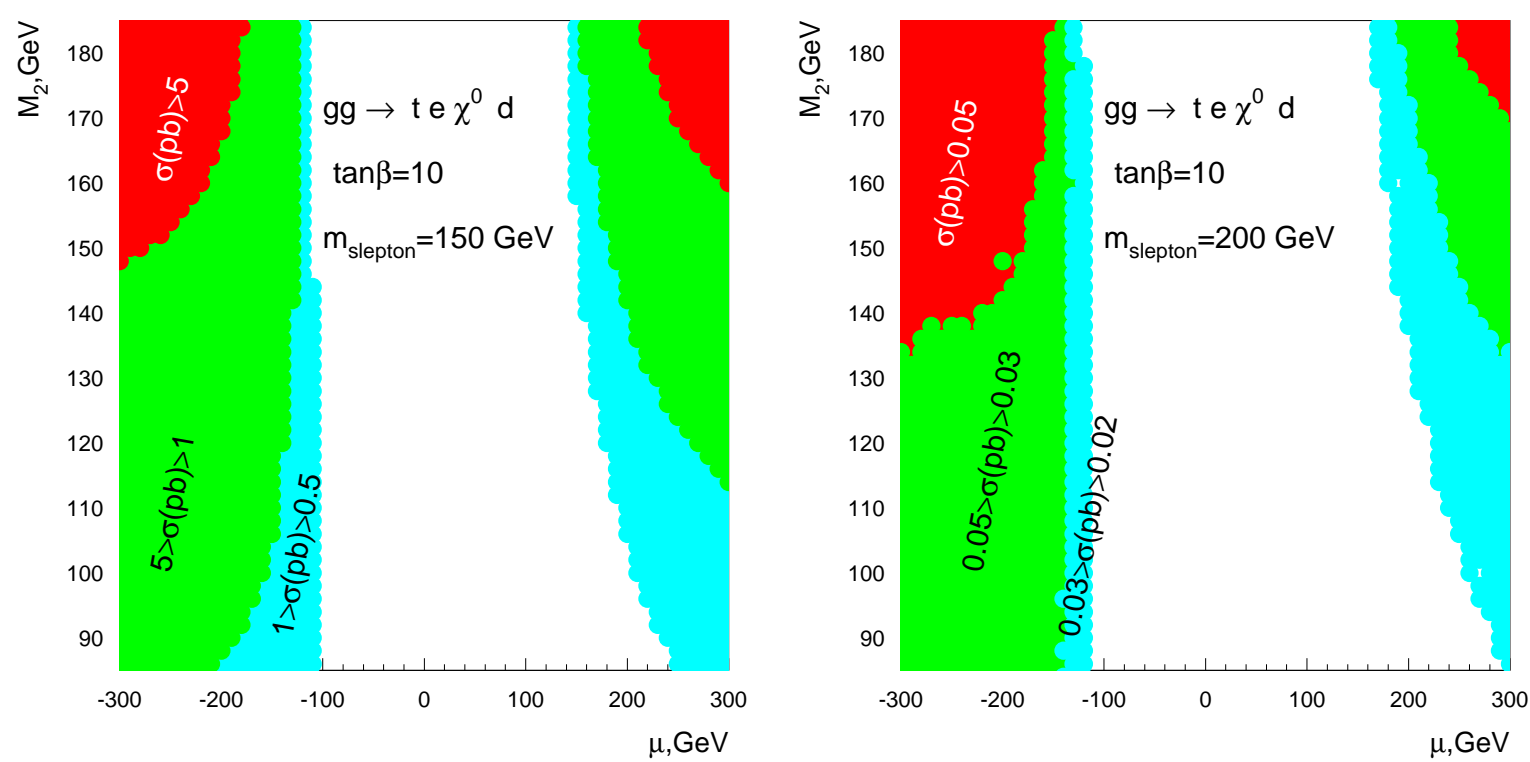

Figure 6. Contours for the cross-section of $g g \rightarrow t \tilde{\chi}^{0} \ell q$ process for $m_{\tilde{\ell}}=150 \mathrm{GeV}$ (left) and $m_{\tilde{\ell}}=200 \mathrm{GeV}$ (right).

$\sharp$ In principle, the neutralino width could be comparable with the width of a slepton, when the $\chi_{1}^{0} \rightarrow \tilde{t} t$ 2-body decay channel becomes open if $m_{\tilde{l}}>m_{\chi}^{0}>m_{t}$. But such case is not relevant to our study since in this scenario the RPV top-quark decay branching ratio will be several orders $(\sim 3-4)$ of magnitude below the sensitivity of the LHC. 


\section{Signatures and details of simulation}

We have used the CompHEP package to generate $t \bar{t}$ events with $R_{p}$ decay of one of the top-quarks $-t \rightarrow \tilde{\chi}^{0} q \ell$ and SM decay of the other one $-\bar{t} \rightarrow W b$. We have studied the cases of hadronic and leptonic decay modes of the $W$-boson from the top-quark undergoing the SM decay. In case of the leptonic decay of $W$, we studied $W \rightarrow \mu \nu$ decay channel in order to enhance the separation between the top $\not R_{p}$ SUSY and SM decay chains.

In the present study we assume that only one $\lambda_{i 3 k}^{\prime}$ parameter in Eq. (3) is nonvanishing. A value of 0.5 for $\lambda_{131}^{\prime}$ was chosen for a reference. The following values of supersymmetric parameters were used: a gaugino mass $M_{2}=120 \mathrm{GeV}$, a higgsino mass parameter $\mu=-300 \mathrm{GeV}, \tan \beta=10$, and a slepton mass either $m_{\tilde{\ell}}=150 \mathrm{GeV}$ $\left(m_{\tilde{\ell}}<m_{\text {top }}\right)$ or $200 \mathrm{GeV}\left(m_{\tilde{\ell}}>m_{\text {top }}\right)$. Under these conditions, the total cross-sections obtained with CompHEP for the $2 \rightarrow 4$ signal process (see Fig. 3. $p_{T}^{q}>10 \mathrm{GeV}$ ) are $8 \mathrm{pb}$ for a slepton mass $m_{\tilde{\ell}}=150 \mathrm{GeV}$ and $480 \mathrm{fb}$ for $m_{\tilde{\ell}}=200 \mathrm{GeV}$. This is to be compared with the total cross section of $833 \mathrm{pb}[1$ for the $t \bar{t}$ background.

With this set of parameters, the mass of the neutralino is $m_{\chi^{0}}=58 \mathrm{GeV}$. If $\lambda_{i 3 k}^{\prime}$ is non-zero only for $i=k=1$, the neutralino will decay into $\bar{\nu}_{e} \bar{b} d$ or $\nu_{e} b \bar{d}(B D N)$ (Fig. (7)).

We also consider the possibility of stop-scharm mixing leading to neutralino decay into $\ell^{-} c \bar{d}$ or $\ell^{+} \bar{c} d$ (Fig. [8). It must be pointed out that such mixing, leading to flavor changing neutral currents (FCNC), is practically not constrained by experimental data [20, 21]. We assume maximum stop-scharm mixing here, but the results can be easily rescaled for any arbitrary value of the mixing. If the stop-scharm mixing is significant, and if one of the stop quarks is lighter than other sfermions appearing in the Feynman diagrams, then the $\tilde{\chi}^{0} \rightarrow \bar{c} d \bar{e}(c \bar{d} e)(C D E)$ channel could be dominant. In this case, one would be able to obtain with much higher efficiency ( in comparison to $B D N$ case ) a narrow peak for the reconstructed top quark mass.

An additional advantage of this decay mode is that the neutralino, by virtue of its Majorana nature, decays equally to positively and negatively charged leptons. This means that half of all events would contain two like-sign leptons. This clean signal signature allows the application of an effective cut to suppress possible backgrounds. The study of the $\not_{p}$ rare top decays at the LHC would also allow the direct measurement of, or the establishment of a limit on stop-scharm mixing. Below, we perform an analysis under both assumptions for neutralino decay: with and without stop-scharm mixing.

PYTHIA 6.2 [22] was used to account for initial and final state radiation and to perform hadronization and decay of resonances. The Lund symmetric fragmentation function has been used for light flavors, but charm and heavier ones - according to the Peterson/SLAC function. The simulation of the signal and background was performed with ATLFAST [23] to take into account the experimental conditions prevailing at LHC for the ATLAS detector 24].

Jet energies were reconstructed by clustering hadronic calorimeters cells of dimensions $\Delta \eta \times \Delta \phi=0.1 \times 0.1$ within the pseudorapidity range $-2.5<\eta<2.5$, where 


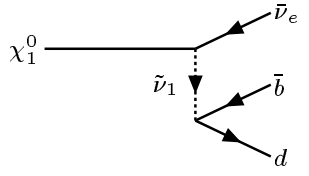

diagr.1

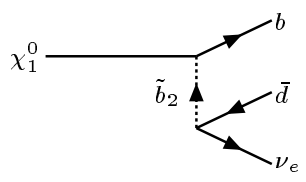

diagr.1

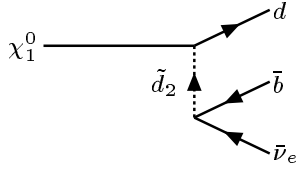

diagr.2

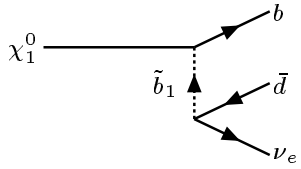

$\operatorname{diagr.2}$

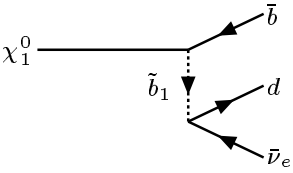

$\operatorname{diagr} .3$

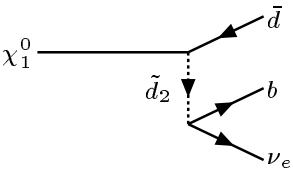

$\operatorname{diagr.3}$

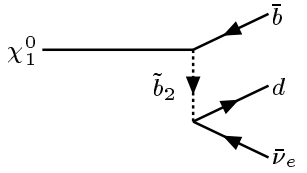

$\operatorname{diagr.4}$

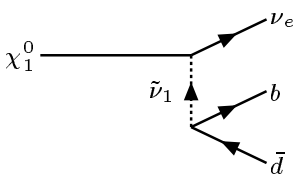

$\operatorname{diagr.4}$

Figure 7. Tree-level diagrams for MSSM $\tilde{\chi}^{0} \rightarrow \bar{\nu}_{e} \bar{b} d\left(\nu_{e} b \bar{d}\right)$ decay.

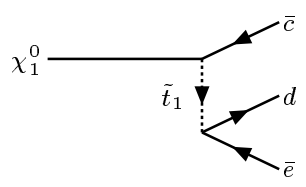

diagr. 1

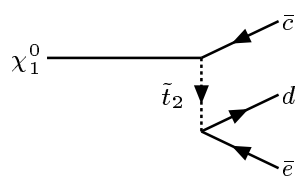

diagr.2

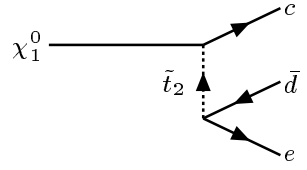

diagr.1

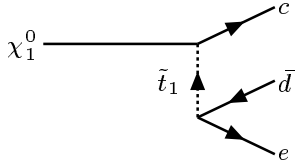

$\operatorname{diagr} .2$

Figure 8. Tree-level diagrams for FCNC $\tilde{\chi}^{0} \rightarrow \bar{c} d \bar{e}(c \bar{d} e)$ decay.

$\phi$ is the azimuthal angle. The hadronic energy resolution of the ATLAS detector is parametrized as $0.5 / \sqrt{E(G e V)} \oplus 0.03$ over this $\eta$ region. Hadronic showers are regarded as jets if its deposited transverse energy $E_{T}$ is greater than $10 \mathrm{GeV}$ within a cone of radius $\Delta R=0.4$. Electromagnetic calorimeters cells of dimensions $\Delta \eta \times \Delta \phi=0.025 \times 0.025$ within the same pseudorapidity range $-2.5<\eta<2.5$ are used to measure the lepton energy. The electromagnetic energy resolution is given by $0.1 / \sqrt{E(G e V)} \oplus 0.007$ over this $\eta$ region. Electromagnetic showers are identified as electron candidates if their $E_{T}>5 \mathrm{GeV}$ within a cone of radius $\Delta R=0.15$. Default ATLFAST electron isolation criteria were applied: separation by $\Delta R>0.4$ from other clusters and $E_{T}<10 \mathrm{GeV}$ deposition in $\Delta R=0.2$ cone around the electron). Default selection criteria for muons ( $P_{T}>6 \mathrm{GeV}$ and $\left.|\eta|<2.5\right)$ and isolation criteria, the same as for in the case of electron, were also applied.

ATLFAST labels a jet as a $b$-jet if a $b$-quark is present in a cone $\Delta R=0.2$ around the reconstructed jet for jets with $\eta<2.5$. Efficiencies for b-jet tagging have been parametrized by $P_{T}$-dependent functions, with maximal saturated efficiency $\epsilon_{b}=0.7$ at high $P_{T}$. We have checked that for slepton mass within the range $100-1000 \mathrm{GeV}$ and $\lambda^{\prime}>0.005$, the lifetime of the neutralino is shorter than the lifetime of a B-meson. 
Therefore displacements of the B-meson vertex due to the neutralino decay will not affect the b-tagging efficiency.

The analysis transverse momentum cuts used for electrons, muons and jets were 10 $\mathrm{GeV}, 10 \mathrm{GeV}$ and $20 \mathrm{GeV}$, respectively.

\section{Signal and background analysis for $\tilde{\chi}^{0} \rightarrow c d e$ channel}

In this section we study $C D E$ neutralino decay channel: $\tilde{\chi}^{0} \rightarrow c d e$. First we analyze kinematical characteristics of jets for this channel. The partonic distributions of the transverse momentum and pseudorapidity of quarks and leptons for the process $p p \rightarrow t \tilde{\chi}^{0} q \ell\left(\tilde{\chi}^{0} \rightarrow c d e\right)$ are shown in Figs. 91 10, 11 and 12, For completeness, we also show distributions for jets originating from $W \rightarrow$ jets decay from top-quark undergoing SM decay mode.
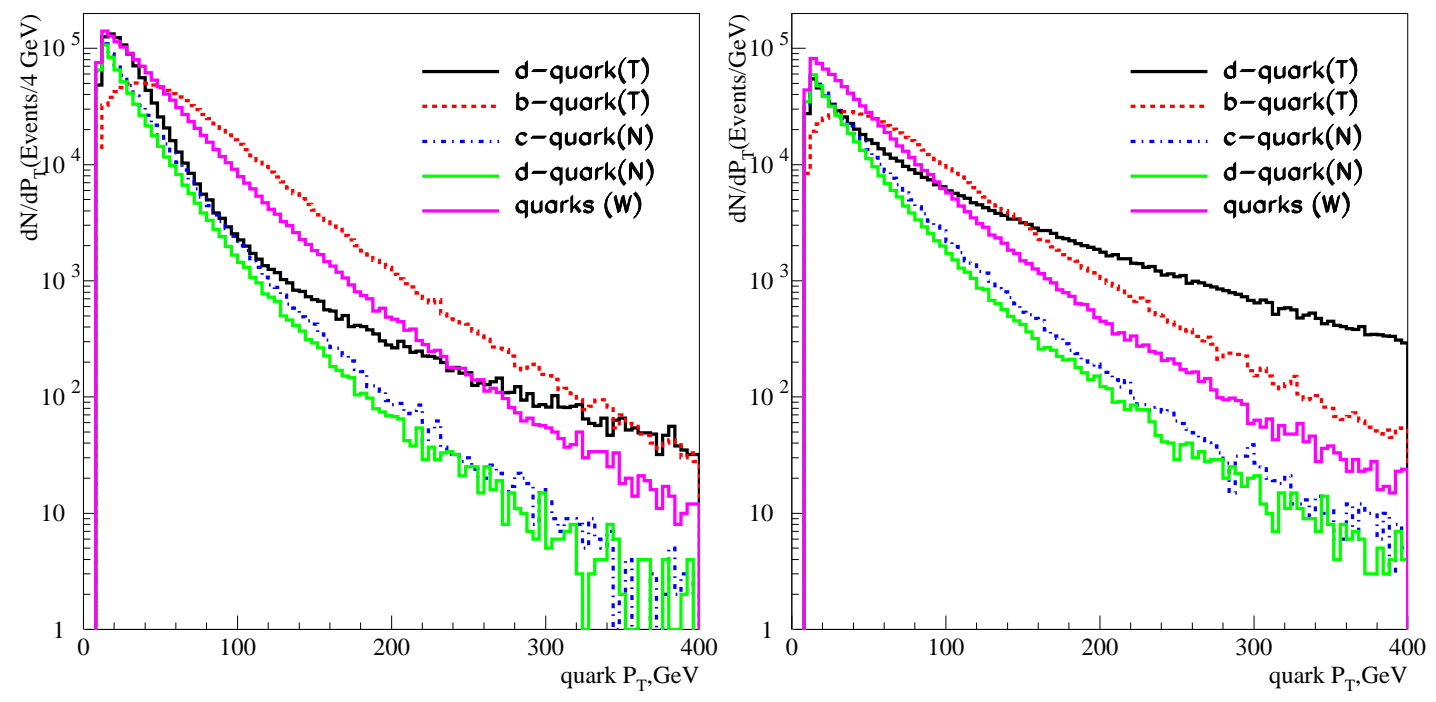

Figure 9. Transverse momentum of quarks from the $p p \rightarrow t \tilde{\chi}^{0} q \ell$ reaction ( $C D E$ channel ) for $m_{\tilde{\ell}}=150 \mathrm{GeV}$ (left) and $m_{\tilde{\ell}}=200 \mathrm{GeV}$ (right ). (T) means a quark originating from a top quark decay, whereas $(\mathrm{N})$ denotes its origin from a neutralino decay and $(\mathrm{W})$ from the $W$ decay.

The transverse momentum spectra of quarks in the process involving a light slepton $\left(m_{\tilde{\ell}}=150 \mathrm{GeV}\right)$ (Fig. Q(left)) indicate that the highest $P_{T}$ jet in the event, with $P_{T}$ $>50 \mathrm{GeV}$ is a $b$-jet from the top quark SM decay. Using $b$-jet tagging technique this jet can be effectively separated from the analysis. Among jets of $P_{T}<50 \mathrm{GeV}$, a $d$-quark from the $\not R_{p}$ top quark decay dominates, as well as in the very high $P_{T}$ region. For the case of a heavy slepton $\left(m_{\tilde{\ell}}=200 \mathrm{GeV}\right)$, a $d$-quark from $\not R_{p}$ decay tends to be with the highest $P_{T}$ in the $P_{T}>150 \mathrm{GeV}$ region and to be emitted in the forward direction (see Fig. 10(right)). The two jets with smallest $P_{T}$ in the events, originate from a neutralino decay.

The transverse momentum for a lepton from $R_{p}$ top quark decay is the largest in the region $P_{T}>50 \mathrm{GeV}$ and $P_{T}>75 \mathrm{GeV}$ for the light and heavy slepton set of events, 


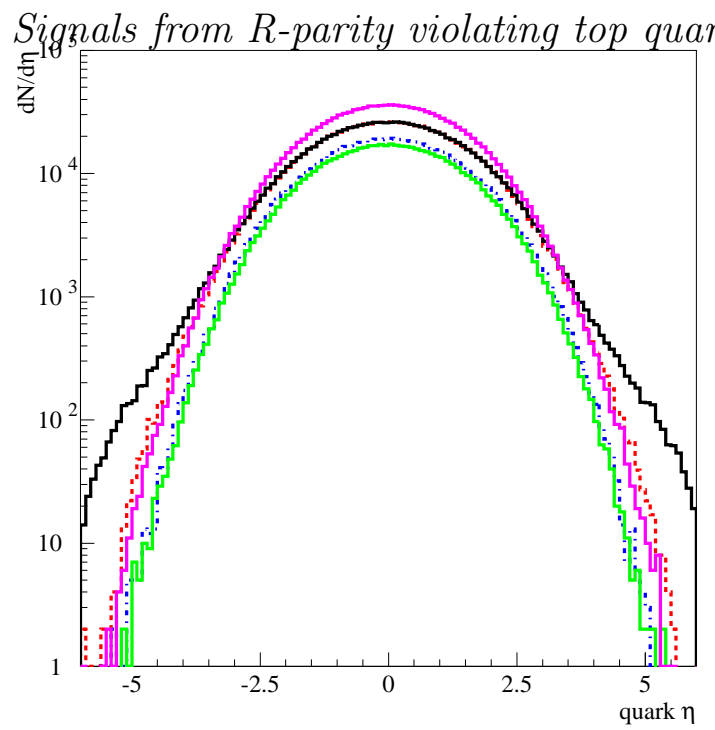

Figure 10. Pseudorapidity of quarks from the $p p \rightarrow t \tilde{\chi}^{0} q \ell$ reaction for $m_{\tilde{\ell}}=150 \mathrm{GeV}$ (left) and $m_{\tilde{\ell}}=200 \mathrm{GeV}$ (right). The notations are the same as in Fig. 9

respectively (see Fig. 11). In terms of the pseudorapidity variable, leptons are mainly emitted in the central region (Fig. 12).
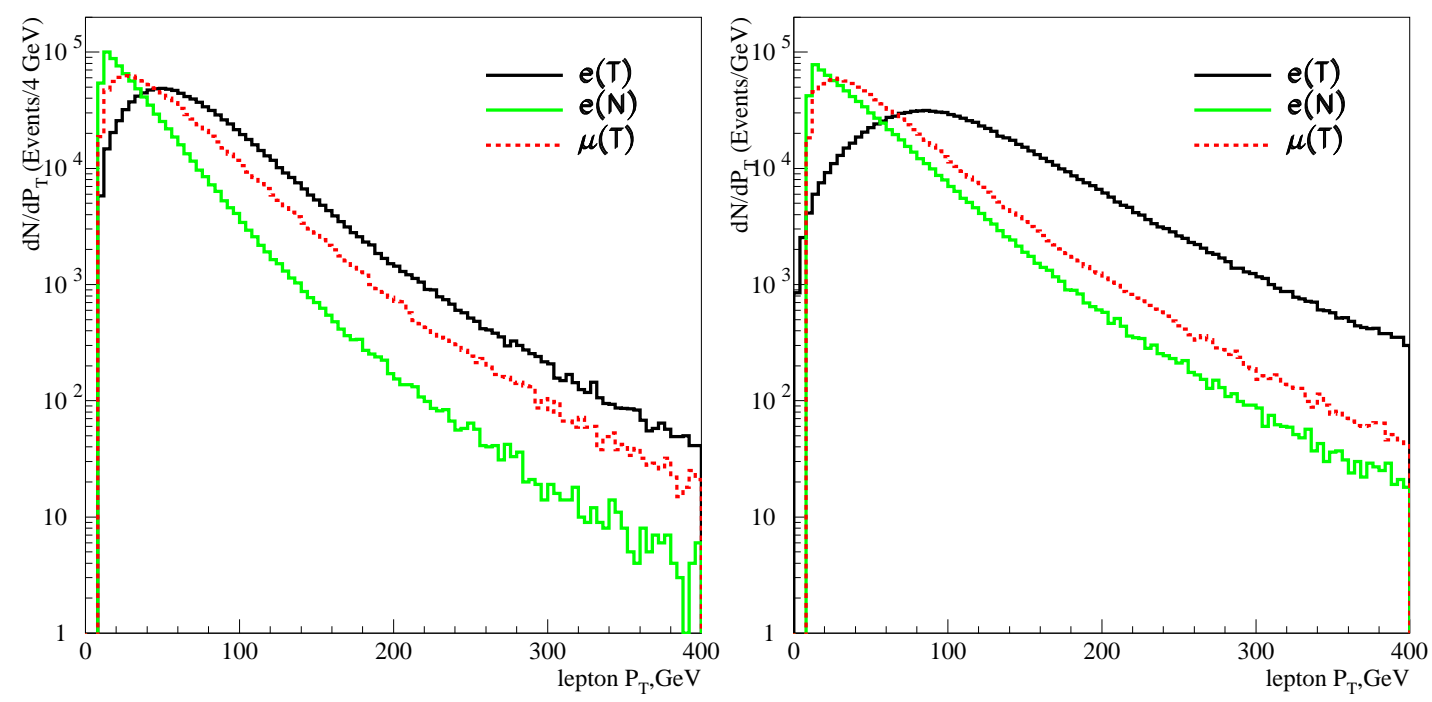

Figure 11. Transverse momentum of leptons from the $p p \rightarrow t \tilde{\chi}^{0} q \ell$ reaction for $m_{\tilde{\ell}}=150 \mathrm{GeV}$ (left) and $m_{\tilde{\ell}}=200 \mathrm{GeV}$ (right). (T) means a lepton originated from the top quark decay, whereas $(\mathrm{N})$ denotes its origin from the neutralino decay and (W) from the $W$ decay.

The effect of initial (ISR) and final state (FSR) QCD radiation approximately doubles the number of jets with $P_{T}>10 \mathrm{GeV}$ produced in the event. Fig. 13] shows the jet multiplicity distribution.

The only non-negligible background for the $C D E$ channel is $t W W(q)$ processes. This process would be a background for the case where $\tilde{\chi}^{0} \rightarrow$ cde when both $W$ 's decay leptonically and the $W$ from the top quark decays hadronically. The Feynman diagrams 


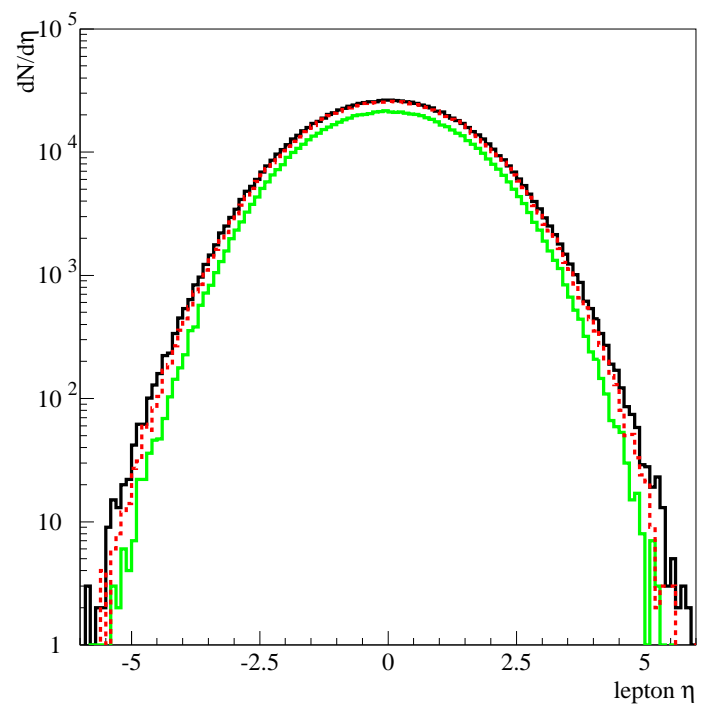

Figure 12. Pseudorapidity of leptons from the $p p \rightarrow t \tilde{\chi}^{0} q \ell$ reaction for $m_{\tilde{\ell}}=150 \mathrm{GeV}$. The notations are the same as in Fig. [11]
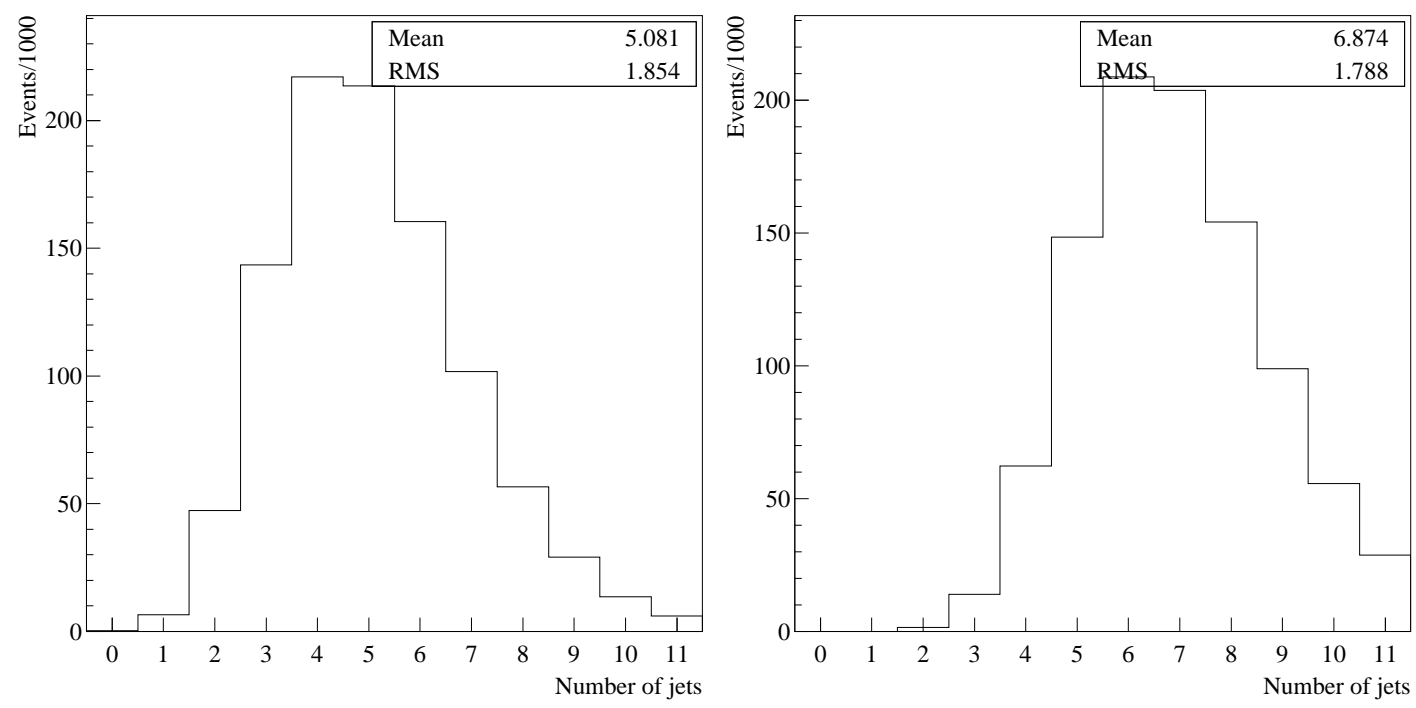

Figure 13. Distribution of the number of reconstructed jets after ATLFAST for the cases of $W \rightarrow \mu \nu$ (left) and $W \rightarrow$ jets.

for $t W W q$ background are shown in Fig. 14. The total cross-section for this process, obtained from CompHEP is $1.1 \mathrm{pb}$.

\section{Results for $\tilde{\chi}^{0} \rightarrow c d e$ channel}

The following cuts have been worked out for the top quark reconstruction:

- At least 4 ( or 6 for $t \rightarrow b j j$ final state) jets with $P_{T}>20 \mathrm{GeV}$ and one electron with $P_{T}>10 \mathrm{GeV}$ within $|\eta|<2.5$ pseudorapidity.

- one jet tagged as a $b$-jet: required to get rid of a $b$-jet from a top quark which 

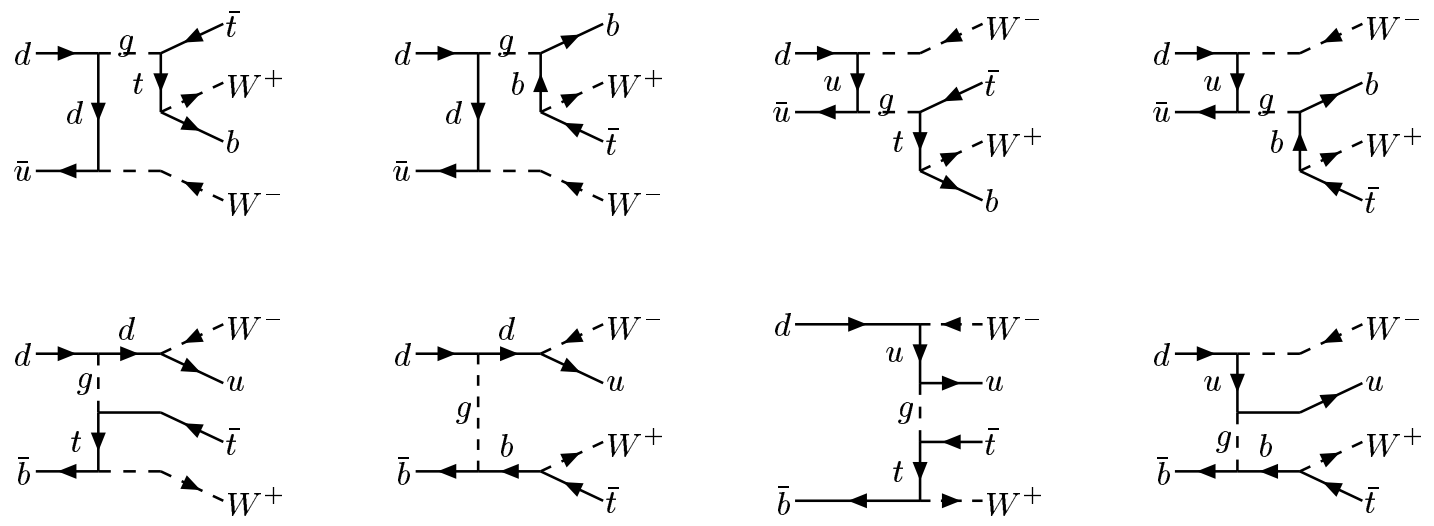

Figure 14. Tree-level diagrams for the $t W W q$ process

decayed in the framework of the SM.

- cut on invariant mass of (jjee), $M($ jjee $)<150 \mathrm{GeV}$ (for the light slepton case) or $<200 \mathrm{GeV}$ (for the heavy slepton case), to suppress the influence of the on-shell slepton on the top quark signal, especially effective in the $m_{\tilde{\ell}}<m_{\text {top }}$ case.

For the mass reconstruction we combine two electrons with the three jets in the event. If the number of jets in the event was larger than three (but less than seven), the three jets with the smallest $P_{T}$ in the event were combined with both electrons.

The resulting mass distributions of the (jjjee) system are shown in Fig. 15 for the two different $W$ decay modes studied and the light slepton case, $m_{\tilde{\ell}}=150 \mathrm{GeV}$ (left), and the heavy one, $m_{\tilde{\ell}}=200 \mathrm{GeV}$ (right), respectively. The data are presented for an integrated luminosity of $100 \mathrm{fb}^{-1}$ (one year of running at high luminosity at LHC) for the light slepton case, and $300 \mathrm{fb}^{-1}$ for the heavy slepton case. After the fiducial cuts given above, the $t W W q$ background for the events with $\tilde{\chi}^{0} \rightarrow c d e$ is appears to be very small and practically is not seen on the Fig. 15.

The events for the heavy slepton case show the shift of the mass value of the top quark signature because of a heavy on-shell slepton mass dominance and big combinatorial jet pairs background.

Based on the kinematical distributions shown above, we define procedures to select and reconstruct the neutralino and the slepton in the events of interest.

For the case of a light slepton, in order to reconstruct the neutralino (Fig. 16, left), we combine the electron with smallest $P_{T}$ with the two jets with smallest $P_{T}$. The figure shows the invariant mass distribution for an integrated luminosity of $100 \mathrm{fb}^{-1}$, with the contributions from events where $W \rightarrow \mu \nu$ and where $W \rightarrow$ jets explicitly indicated.

For the heavy slepton case (Fig. 16, right) we use the same procedure, but due to a lower production cross-section, we present the mass reconstruction for an integrated luminosity of $300 \mathrm{fb}^{-1}$.

For the reconstruction of the slepton, we combine both electrons in the event with the same two soft jets (Fig. 17). 

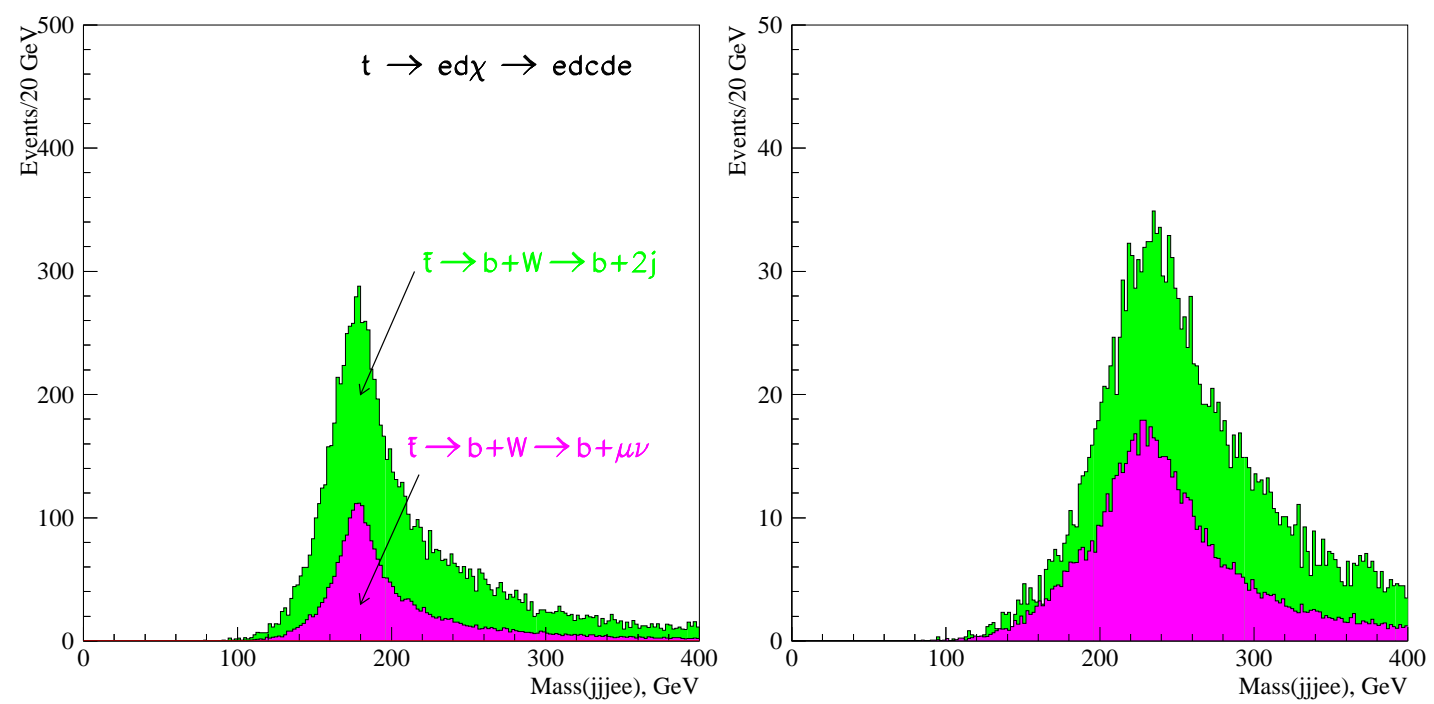

Figure 15. Detector level invariant mass distribution for the (jjjee) system in $C D E$ channel and light (left) or heavy (right) slepton cases (see text). The distributions are normalized for an integrated luminosity of $100 \mathrm{fb}^{-1}$ and $300 \mathrm{fb}^{-1}$, respectively.
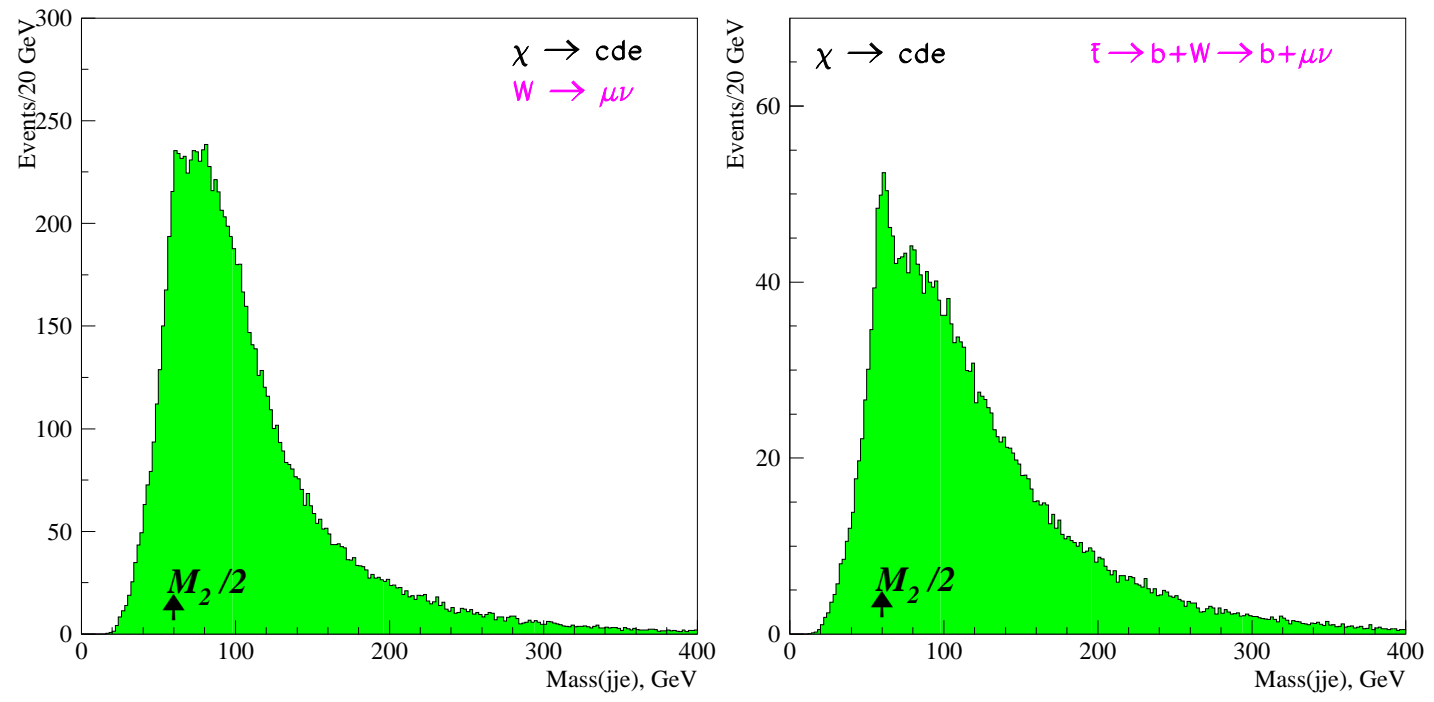

Figure 16. Detector level invariant mass distribution for the (jje) system for $C D E$ channel and the light slepton (left) or the heavy slepton ( right) cases. The distributions are normalized for an integrated luminosity of $100 \mathrm{fb}^{-1}$ and $300 \mathrm{fb}^{-1}$, respectively.

Light slepton case presented in Fig. 177 (left).

For the heavy slepton case (Fig. 17, right) we use the same procedure, but again due to a lower production cross-section, we present the mass reconstruction of sleptons for an integrated luminosity of $300 \mathrm{fb}^{-1}$. 

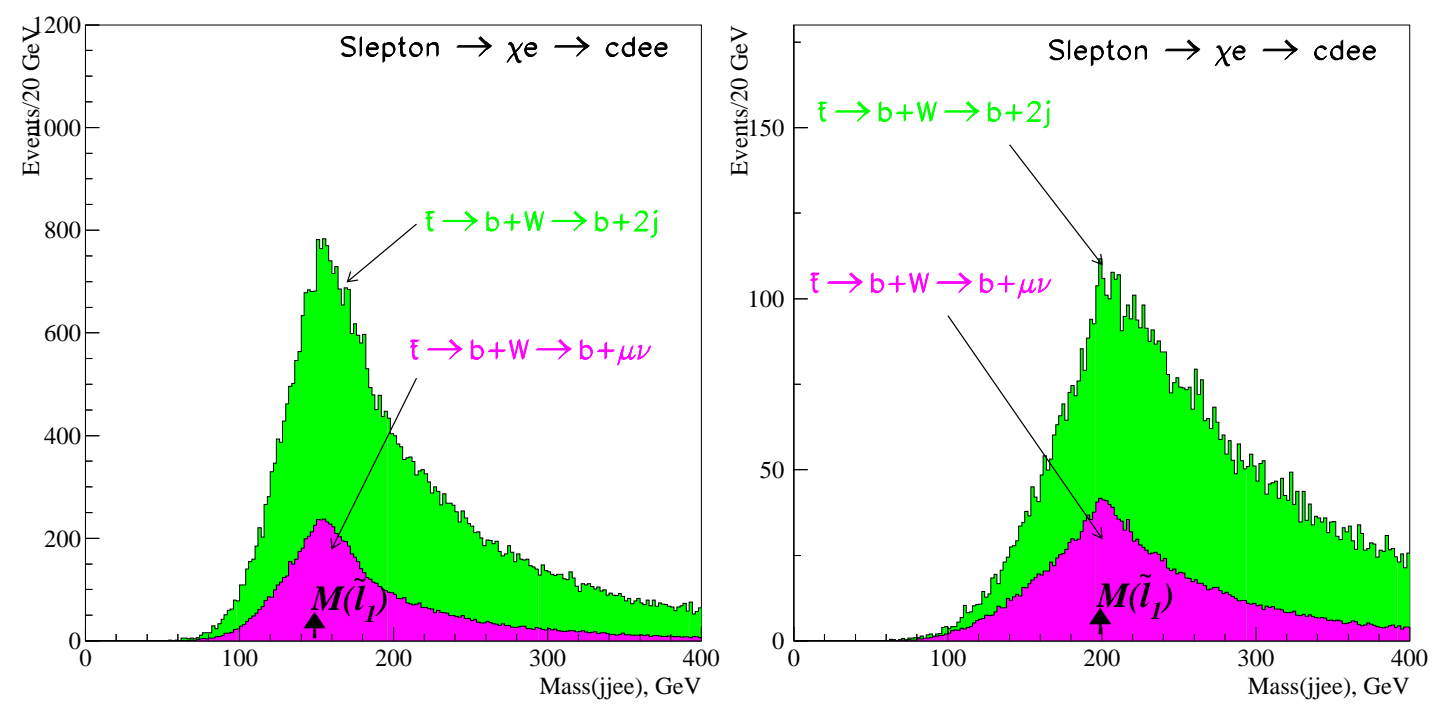

Figure 17. Detector level invariant mass distribution for the (jjee) system for $\tilde{\chi}^{0} \rightarrow c d e$ and the light slepton (left) or the heavy slepton ( right) cases. The distributions are normalized for an integrated luminosity of $100 \mathrm{fb}^{-1}$ and $300 \mathrm{fb}^{-1}$, respectively.

\section{Results for $\tilde{\chi}^{0} \rightarrow b d \nu$ channel}

In the case where $\tilde{\chi}^{0} \rightarrow b d \nu$, we considered only the scenario where $W$ decays into jets, since the decay mode $W \rightarrow \nu \ell$ leads to two neutrinos in the final state, making the event reconstruction difficult. Fig. 18 shows the partonic distributions of the transverse momentum of quarks for events where the neutralino decays $\tilde{\chi}^{0} \rightarrow b d \nu$ (right).

Thus, we are led to event topologies with 6 quarks, one electron and a neutrino at the parton level. Although the large combinatorial background in jet pair selection makes it difficult to reconstruct the signal for the event under study, one can observe certain correlations in these distributions. For example, the two softest light jets are originating from a neutralino.

The natural background for the case where $\tilde{\chi}^{0} \rightarrow b d \nu$ is $t \bar{t}$ and, possibly, $t \bar{t} b \bar{b}$ production. These backgrounds have the topology of the signal when one $W$-boson decays into jets and the other $W$ decays leptonically to $e \nu$.

We applied the following selection cuts for reconstructing events where one top quark decays via the $R_{p}$ SUSY mechanism, and its decay product, a neutralino, decays to $b d \nu$, while the other top quark in the event, decays in the SM to a $b$ quark and two jets.

- at least 6 jets with $P_{T}>20 \mathrm{GeV}$ and one electron with $P_{T}>40 \mathrm{GeV}$ and $|\eta|<2.5$ (see Fig 19).

- at least two jets tagged as b-jets with $P_{T}>20 \mathrm{GeV}$ : one for the top quark with the SM decay and another from the decay of the neutralino.

- No two-jet combination should have the invariant mass of a $W$, in a window \pm 20 


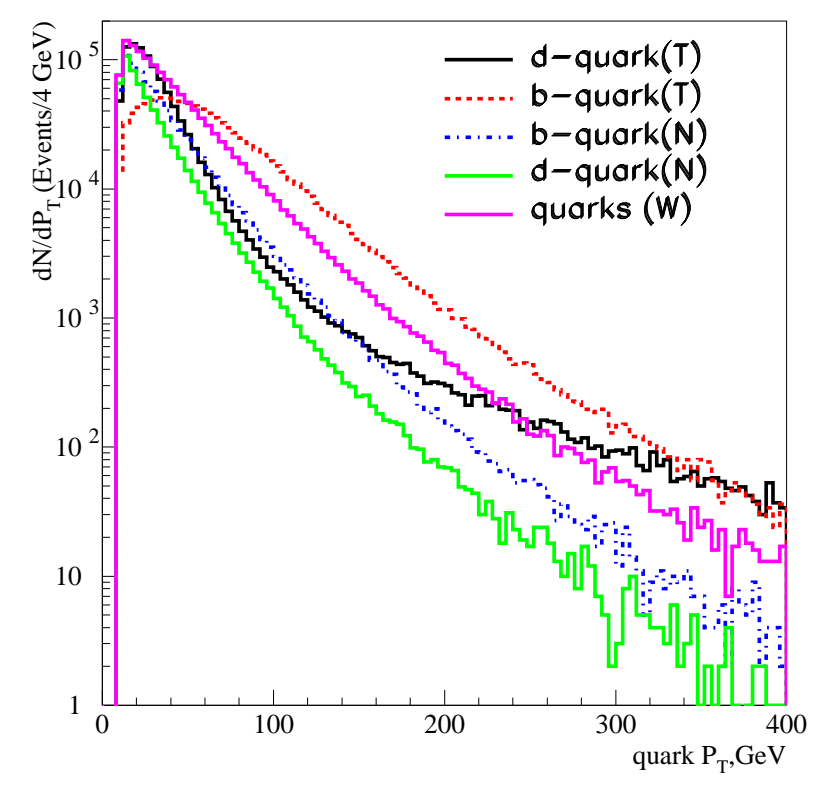

Figure 18. Transverse momentum of quarks from the $p p \rightarrow t \tilde{\chi}^{0} q \ell$ reaction for $m_{\tilde{\ell}}=150 \mathrm{GeV}$ where $\tilde{\chi}^{0} \rightarrow b d \nu$. (T) means a quark originated from a top quark decay, whereas $(\mathrm{N})$ denotes its origin from a neutralino decay and $(\mathrm{W})$ from a $W$ decay.

$\mathrm{GeV}$.

- cut on the mass of the system $(e b \nu)<140 \mathrm{GeV}$ (see Fig 19), to suppress the $t \bar{t}$ background, since no such decays of the top quark should occur in the $\not_{p}$ top decay scenario.

- cut on the transverse mass of $(j j e \nu): M\left(j j e E_{T}\right)<150 \mathrm{GeV}$ (for the light slepton case ) to suppress the influence of the on-shell slepton on the top quark signal, especially effective in the $m_{\tilde{\ell}}<m_{\text {top }}$ case.

The mass reconstruction was performed for one electron, $E_{T}$ and three jets in the event. When the number of jets in the event was larger than three (but less than seven), the three jets with the smallest $P_{T}$ in the event were taken into the reconstruction system.

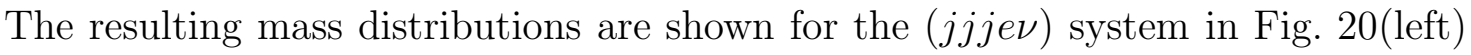
for $\tilde{\chi}^{0} \rightarrow b d \nu$ decay channel and the light slepton $\operatorname{case}\left(m_{\tilde{\ell}}=150 \mathrm{GeV}\right)$. The data are presented for an integrated luminosity of $100 \mathrm{fb}^{-1}$. The distributions for signal and background are shown with separate contributions from $t \bar{t}$ and $t \bar{t} b \bar{b}$ events. The generation of the $t \bar{t} b \bar{b}$ background has been performed with CompHEP and the crosssection obtained was $5.44 \mathrm{pb}$. As one can see below, this background brings contribution of about $2 \%$ to the total background for all signal signatures considered.

For the heavy slepton case $\left(m_{\tilde{\ell}}=200 \mathrm{GeV}\right)$ we present our results in Fig. 20(right)for the signal events only for an integrated luminosity of $300 \mathrm{fb}^{-1}$. Bearing in mind the large $t \bar{t}$ background for $\tilde{\chi}^{0} \rightarrow b d \nu$, it is clear that no signal can be extracted 

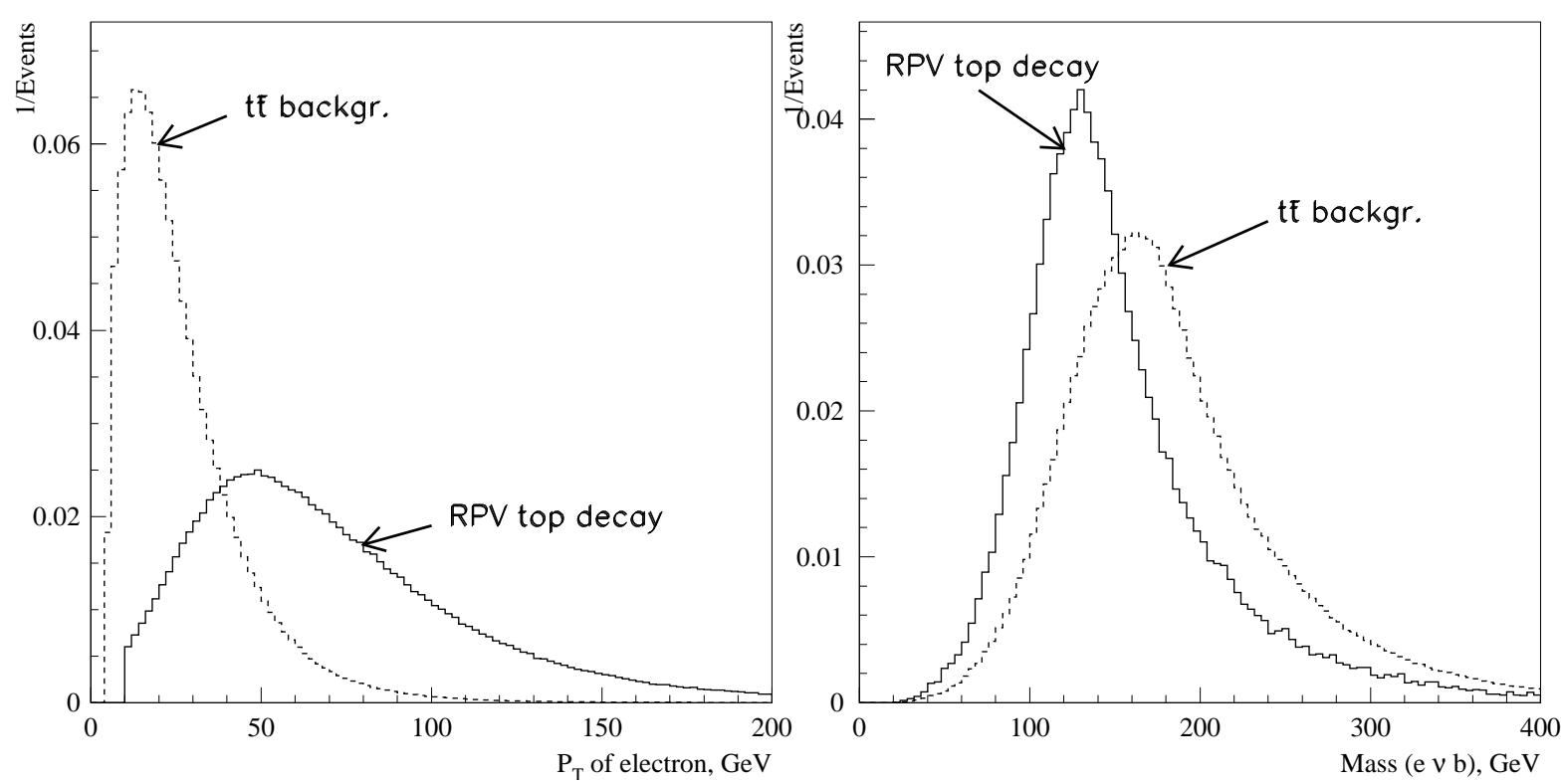

Figure 19. Parton level transverse momentum of electron produced in $R_{p}$ top decay (solid line) and in $W$-boson leptonic decay in $t \bar{t}$ events (dashed line) (left); Detector

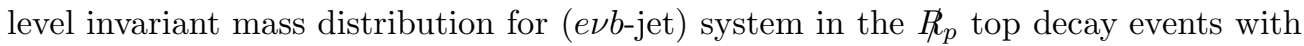
a neutralino decay to $b d \nu$ (solid line) and $t \bar{t}$ events (dashed line )(right).

for the $B D N$ channel and the heavy slepton case. We do not show for this case the distributions for backgrounds due to their overwhelming dominance.
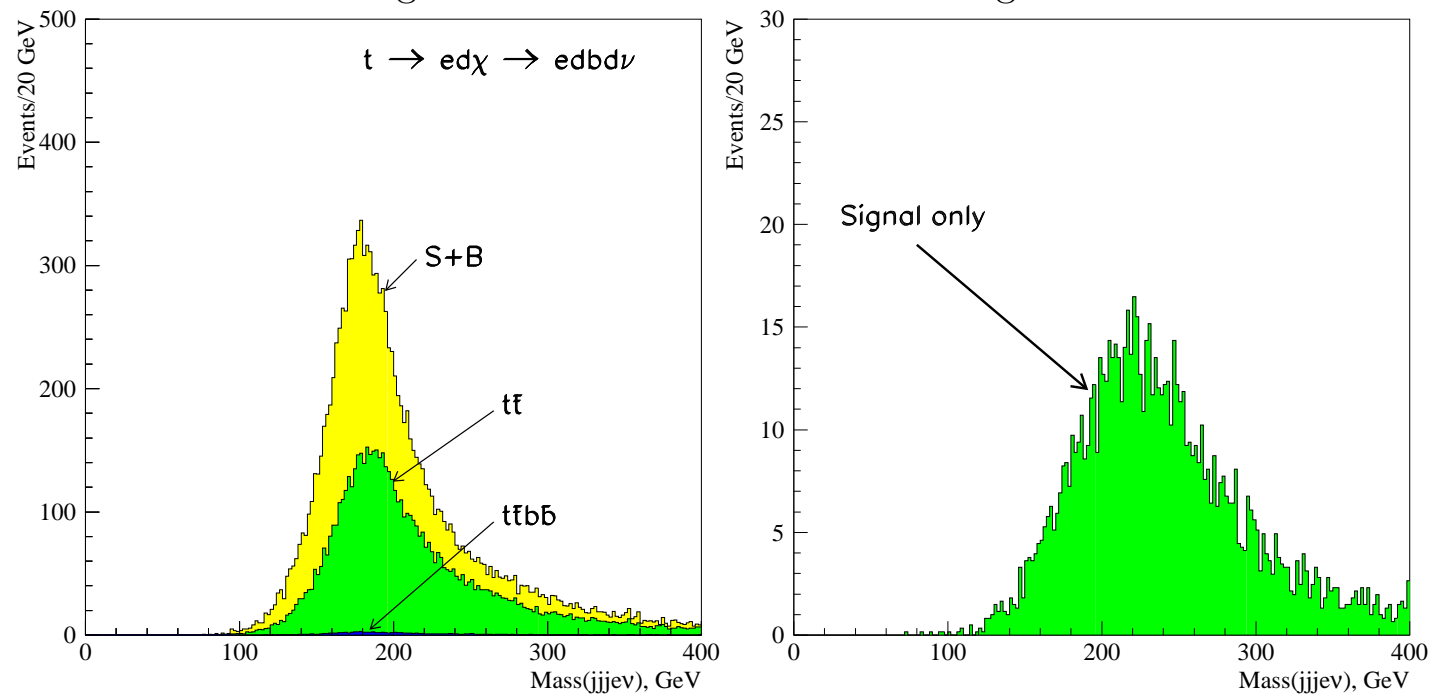

Figure 20. Detector level invariant mass distribution for the $(j j j e \nu)$ system for $m_{\tilde{\ell}}=150 \mathrm{GeV}$ (left) and $m_{\tilde{\ell}}=200 \mathrm{GeV}$ (right) cases for $\tilde{\chi}^{0} \rightarrow b d \nu$ decay channel. Simulations were done for an integrated luminosity of $100 \mathrm{fb}^{-1}$ and $300 \mathrm{fb}^{-1}$, respectively. The background of $t \bar{t}$ and $t \bar{t} b \bar{b}$ production for $m_{\tilde{\ell}}=150 \mathrm{GeV}$ case is presented.

For these events to reconstruct the neutralino, we combine the $\#_{T}$ with the two jets with smallest $P_{T}$, and for the slepton, we combine the same two jets with the softest 
electron and the $\mathbb{E}_{T}$. The shift from nominal simulated values for the neutralino ( 60 $\mathrm{GeV}$ ) and the slepton ( $150 \mathrm{GeV}$ ) is due to the difficulties to calculate the energy of neutrino.
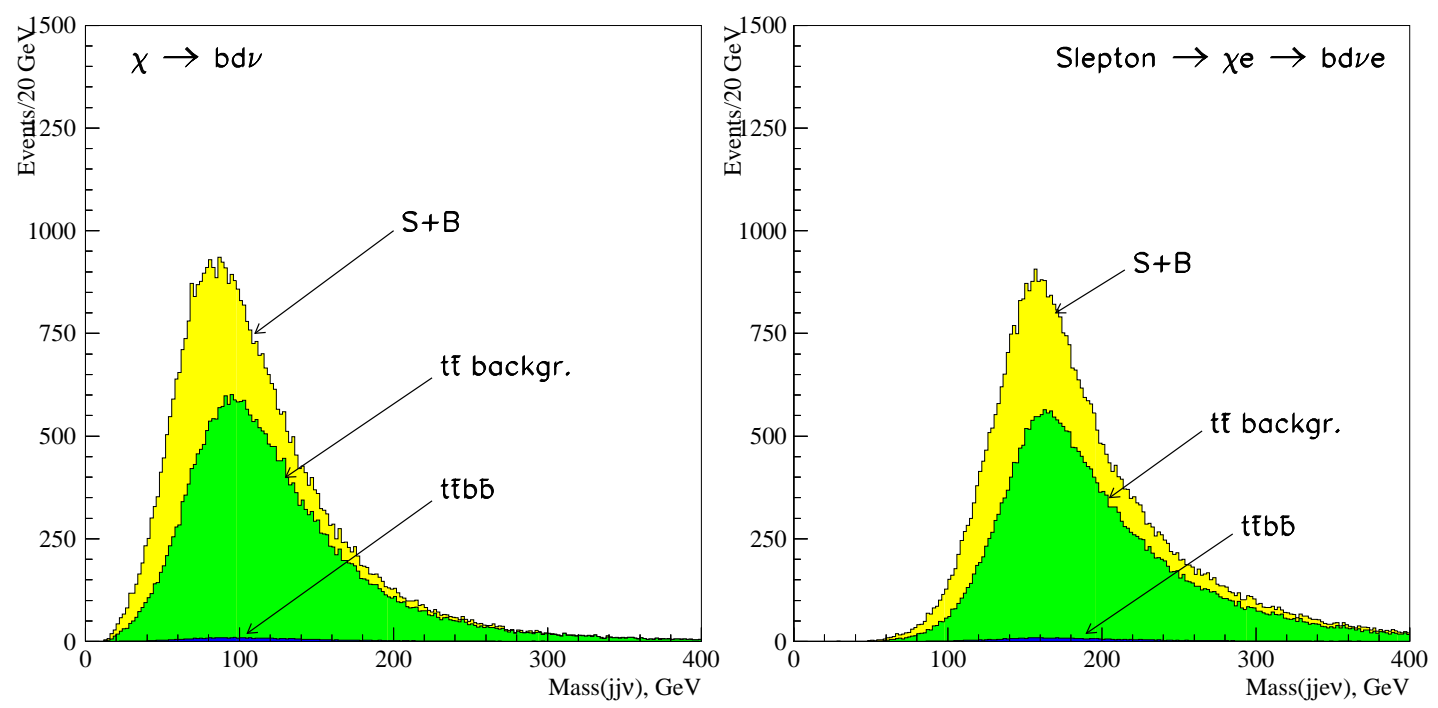

Figure 21. Detector level invariant mass distribution for the $(j j \nu)$ (left) and $(j j e \nu)$ (right) systems for the case $m_{\tilde{\ell}}=150 \mathrm{GeV}$ and $\tilde{\chi}^{0} \rightarrow b d \nu$. Background of $t \bar{t}$ events is also shown. The distributions are normalized for an integrated luminosity of $100 \mathrm{fb}^{-1}$.

The relative strengths of signal $\left(\tilde{\chi}^{0} \rightarrow b d \nu\right)$ and the $t \bar{t}$ background are compared in Figs. 20(left) and 21].

For the heavy slepton case we use the same procedure, but due to a lower production cross-section the signal cannot be seen due to the $t \bar{t}$ background dominance.

It is worth to mention that the accuracy of the $t \bar{t}$ background measurement for the $B D N$ decay channel would be very important. An error of $1 \%$ on the top quark mass measurements corresponds to an error of $5 \%$ on the total ttbar cross-section, or vice versa. Systematic uncertainties of the measurements of the top quark mass are dominated by the jet energy scale and FSR effects. Assuming a 1\% scale uncertainty, which is feasible in ATLAS, and varying the jet cone size, should reduce the total systematic uncertainties[?]. The precision of the measurements of the total $t \bar{t}$ crosssection is expected also to be dominated by the knowledge of the absolute scale of the luminosity. In any case, the significant amount of data will be required before the $t \bar{t}$ background contribution can be estimated accurately.

\section{1. $L H C$ reach}

Our final results are presented in Tables 1, 2 and 3 and Fig. 22.

Tables 10and 2] present signal and background cut efficiencies $\left(\epsilon^{C D E}\right.$ and $\left.\left.\epsilon^{B D N}\right)\right)$ for the channels $\tilde{\chi}^{0} \rightarrow c d e$ and $\tilde{\chi}^{0} \rightarrow b d \nu$ channels, respectively, for the light slepton case. The cuts were optimized such that $\epsilon_{\text {signal }}^{B D N} / \epsilon_{\text {background }}^{B D N} \simeq 12$, while $\epsilon_{\text {signal }}^{C D E} / \epsilon_{\text {background }}^{C D E} \simeq$ 
7.1(5.6) for leptonic (hadronic) decay channels for $W$-boson. For the heavy slepton case the $\epsilon_{\text {signal }}^{C D E} / \epsilon_{\text {background }}^{C D E}$ ratio is similar to the one for the light slepton case.

Table 1. Effective cuts and respective efficiencies $(\epsilon(\%))$ for the signal events with $\tilde{\chi}^{0} \rightarrow c d e$.

\begin{tabular}{lcccc} 
& $\tilde{\chi}^{0} \rightarrow c d e$ & $t \rightarrow W W j$ & $\tilde{\chi}^{0} \rightarrow c d e$ & $t \rightarrow W W j$ \\
& $W \rightarrow \mu \nu$ & $\mathrm{BG}$ & $W \rightarrow 2$ jets & $\mathrm{BG}$ \\
\hline $\begin{array}{l}\text { Number of events } \\
\mathrm{L}=100 \mathrm{fb}^{-1}\end{array}$ & & & & \\
\hline Cuts used: & $\epsilon 6012$ & 67 & 538369 & 417 \\
\hline 2 leptons & 49.46 & 59.21 & 47.93 & $5(\%)$ \\
\hline 2 leptons +4 jets & 36.29 & 21.50 & 17.98 & 8.61 \\
\hline 1 b-jet tag & 19.46 & 9.65 & 9.40 & 3.44 \\
\hline $\mathrm{M}_{\text {slep }}<150 \mathrm{GeV}$ & 3.53 & 0.50 & 1.90 & 0.34
\end{tabular}

Table 3 gives the number of expected events, calculated for an integrated luminosity of $L=100 \mathrm{fb}^{-1}$, for the channels $C D E$ and $B D N$ and for the light slepton mass $m_{\tilde{\ell}}=150 \mathrm{GeV}$. For the heavy slepton case, i.e. $m_{\tilde{\ell}}=200 \mathrm{GeV}$ we only present in parentheses the numbers only for the $C D E$ case, since the $B D N$ case is hopeless in view of the dominant $t \bar{t}$ background.

As can be seen from this table, the $C D E$ signature has negligible background for both hadronic and leptonic $W$ decay channels. The $B D N$ channel, on the contrary, has much larger background coming from SM $t \bar{t}$ events. Nevertheless, the $t \bar{t}$ is effectively suppressed by the fiducial cuts, and the $S / \sqrt{B} \sim 50$ for the case of a light slepton.

Table 2. Effective cuts and respective efficiencies $(\epsilon(\%))$ for the signal events with $\tilde{\chi}^{0} \rightarrow b d \nu$.

\begin{tabular}{lccc} 
& $\tilde{\chi}^{0} \rightarrow b d \nu$ & $t \bar{t}$ & $t \bar{t} b \bar{b}$ \\
& $W \rightarrow 2 j e t s$ & $\mathrm{BG}$ & $\mathrm{BG}$ \\
\hline Number of events at & & & \\
$\mathrm{L}=100 \mathrm{fb}^{-1}$ & 538576 & 6100000 & 535000 \\
\hline Cuts used: & $\epsilon(\%)$ & $\epsilon(\%)$ & $\epsilon(\%)$ \\
\hline Cuts used: & & & \\
\hline 1 lepton & 61.81 & 45.53 & 48.08 \\
\hline 6 jets +1 lepton & 24.22 & 5.34 & 30.88 \\
\hline 2 b-jet tags & 7.17 & 2.17 & 10.72 \\
\hline $\mathrm{M}_{(\text {evb })}<140 \mathrm{GeV}$ & 3.57 & 0.53 & 2.00 \\
\hline $\mathrm{M}_{\text {slep }}<150 \mathrm{GeV}$ & 1.12 & 0.09 & 0.30
\end{tabular}


Table 3. Number of expected signal events for $C D E\left(\tilde{\chi}^{0} \rightarrow c d e\right)$ and $B D N\left(\tilde{\chi}^{0} \rightarrow b d \nu\right.$ channels for an integrated luminosity of $L=100 \mathrm{fb}^{-1}$. Background(BG) presented are: $t W W q$ events for $\tilde{\chi}^{0} \rightarrow c d e$ case and $t \bar{t}+t \bar{t} b \bar{b}$ production for $\tilde{\chi}^{0} \rightarrow b d \nu$ sample of signal events. Heavy slepton case for the $C D E$ channel is given in parenthesis.

\begin{tabular}{cccccccc}
\hline Signal & $\begin{array}{c}\text { Mass } \\
\text { window } \\
(\mathrm{GeV})\end{array}$ & $\begin{array}{c}\text { Signal } \\
\text { events }\end{array}$ & BG & $\begin{array}{c}\text { Signal } \\
\text { events }\end{array}$ & BG & $\begin{array}{c}\text { Signal } \\
\text { events }\end{array}$ & BG \\
& & & & & & \\
\hline & & $\tilde{\chi}^{0} \rightarrow c d e$ & & $\tilde{\chi}^{0} \rightarrow c d e$ & & $\tilde{\chi}^{0} \rightarrow b d \nu$ & \\
$W \rightarrow \mu \nu$ & & $W \rightarrow 2 j$ & & $W \rightarrow 2 j$ & \\
\hline top quark & $175 \pm 10$ & $949(-)$ & $0.1(-)$ & $2526(-)$ & $0.3(-)$ & 1683 & 1150 \\
\hline & $175 \pm 20$ & $1535(-)$ & $0.1(-)$ & $4389(-)$ & $0.5(-)$ & 2954 & 2138 \\
\hline neutralino & $175 \pm 30$ & $1876(-)$ & $0.1(-)$ & $5588(-)$ & $0.7(-)$ & 3835 & 2882 \\
\hline & $60 \pm 10$ & $1991(88)$ & $0.2(0.5)$ & $6738(182)$ & $0.9(0.9)$ & 3415 & 2551 \\
\hline selectron & $60 \pm 20$ & $3602(149)$ & $0.4(0.9)$ & $12433(294)$ & $1.8(1.6)$ & 6346 & 5172 \\
\hline & $150 \pm 30$ & $4906(199)$ & $0.6(1.3)$ & $17325(390)$ & $2.7(2.4)$ & 8558 & 7874 \\
\hline & $150 \pm 30$ & $3985(141)$ & $0.4(0.7)$ & $13208(375)$ & $1.5(1.8)$ & 6253 & 8079 \\
\hline & $150 \pm 40$ & $6278(240)$ & $0.7(1.4)$ & $21786(677)$ & $2.9(3.5)$ & 10112 & 13969
\end{tabular}

The sensitivity of the LHC to $\lambda^{\prime}$ mainly depends on the slepton mass, $\mu, M_{2}$ and $\tan \beta$ which define the top $R_{p}$ decay branching ratio. For our particular choice of these parameters, we estimate the potential of the LHC to measure $\lambda^{\prime}$ coupling by studying the significance $S / \sqrt{B}$ versus $\lambda^{\prime}$ for both cases of neutralino decay presented in Fig. 22.
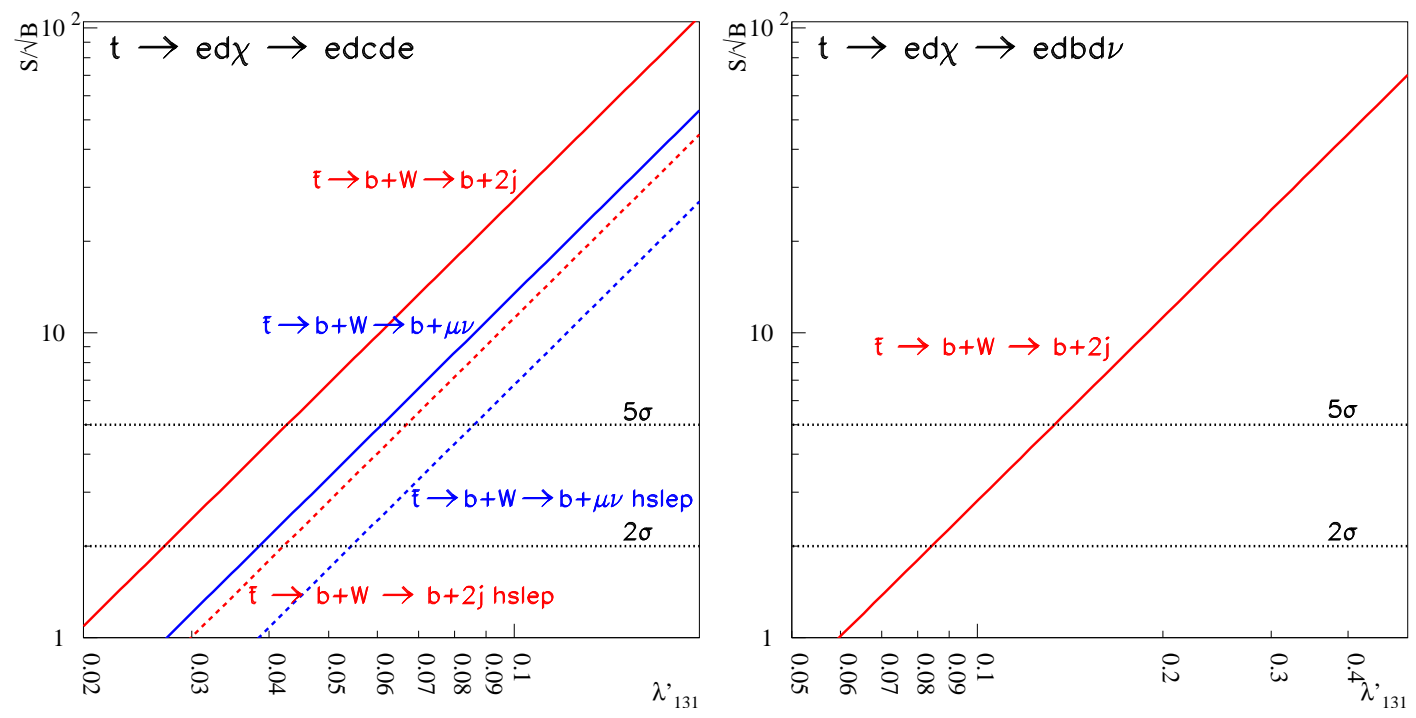

Figure 22. $S / \sqrt{B}$ vs $\lambda^{\prime}$ for $C D E$ (left) and $B D N$ (right) channels. For $W \rightarrow \mu \nu$ decay channel the data are presented for events with $m_{\tilde{\ell}}=150 \mathrm{GeV}$ ( solid line ) and $m_{\tilde{\ell}}=200 \mathrm{GeV}$ ( dashed line ). 
One can see the apparent advantage of the FCNC channel $\tilde{\chi}^{0} \rightarrow c d e$ which allows the determination of the exclusion limits on $\lambda^{\prime}>0.006\left(\lambda^{\prime}>0.03\right)$ at $2 \sigma$ CL or even the possibility to observe the signal at $5 \sigma$ CL for $\lambda^{\prime}>0.01\left(\lambda^{\prime}>0.05\right)$ for light (heavy) slepton case. The case with the SM top $\rightarrow b j j$ is more promising since it provides more statistics. One should remember that we have assumed the maximal stop-scharm mixing $\left(V_{\tilde{c} \tilde{t}}\right)$ in our analysis. Therefore the limit on $\lambda^{\prime}$ presented above should be understood as the limit on $\lambda^{\prime} \times V_{\tilde{c} \tilde{t}}$.

For the $B D N$ channel, the sensitivity to $\lambda^{\prime}$ is significantly reduced: $\lambda^{\prime}>0.15$ at $2 \sigma$ CL for the light slepton case. For the heavy slepton case the $B D N$ channel is not observable.

The limit on $\lambda^{\prime}$ depends on several SUSY parameters. To reduce the model dependence, it is much more convenient to present the results in terms of limits on the branching fraction of $\not R_{p}$ top quark decay $-B F_{\not h_{p}}^{t}=B F\left(t \rightarrow q \chi^{0} \bar{\ell}\right)$. For the light slepton case, the LHC is sensitive to $B F_{R_{p}}^{t} \tilde{>} 3 \times 10^{-6}$ for the $C D E$ channel and to $B F_{R_{p}}^{t} \tilde{>} 2 \times 10^{-3}$ for the $B D N$ channel at $2 \sigma \mathrm{CL}$.

In the case of a heavy slepton scenario, the LHC is sensitive to $B F_{R_{p}}^{t} \tilde{>} 3 \times 10^{-8}$. It looks strange that the LHC is much more sensitive to $B F_{R_{p}}^{t}$ in the heavy slepton case. The puzzle is hidden in the formal definition of $B F_{P_{p}}^{t}=B F\left(t \rightarrow q \chi^{0} \bar{\ell}\right)$ which assumes that $B F_{R_{p}}^{t}$ is calculated for the on-mass-shell top-quark. However, for the heavy slepton case there are strong off-mass-shell top-quark effects and, therefore, it is more convenient to define $B F_{R_{p}}^{t}$ as a ratio of $R_{p}$ and SM cross sections of the topquark production and decay. For this definition, light- and heavy-slepton case limits are consistent with each other and for heavy slepton one has the $B F_{R_{p}}^{t}$ limit of about $5 \times 10^{-6}$ i.e. a little bit worse than for the light slepton scenario.

It is a quite generic and quasi model independent fact that the sensitivity of the LHC to $B F_{R_{p}}^{t}$ is of the order of $10^{-6}$ for $C D E$ channel while for $B D N$ it is about three orders of magnitude worse.

\section{Conclusions}

We have studied the sensitivity of the CERN LHC collider to rare $\mathbb{R}_{p}$ top quark decay. We have shown that it is very important to consider top quark production and its $R_{p}$ decay together (approach (iii) defined in Sect. 2) in order to obtain the correct cross section rates and relevant distributions. The necessity of this treatment is related to the fact that the $\not_{p}$ top quark decay involves a heavy intermediate sfermion which forces the top quark to be off-shell.

Our studies have been done at the detector fast simulation level. We have shown that the LHC collider offers a unique potential to study rare top quark decays within the framework of supersymmetry with broken $R$-parity with a sensitivity on the branching 
fraction $B F_{R_{p}}^{t}$ of the R-parity violating top quark decay in the range of $\simeq 10^{-6}-10^{-7}$ for the $C D E$ channel and $\simeq 10^{-3}$ for the $B D N$ channel.

We have shown that the $C D E$ channel is not only very sensitive to the $\lambda^{\prime}$ coupling, but will also allow the measurement (or the setting of a limit) on the $\tilde{c}-\tilde{t}$ mixing which is practically unconstrained, at present. The $B D N$ channel can provide an independent limit on the $\lambda^{\prime}$ coupling, irrespective of the squark-mixing parameter.

\section{Acknowledgments}

We would like also to thank G. Azuelos, H.Baer, D.Froidevaux, F. Gianotti, S. Heywood, I. Hinchliffe, M.Smizanska, F.Paige, G. Polesello, for their comments about the subject. M-H.G., C.L. and R.M. thank NSERC/Canada for their support. This research was supported in part by the U.S. Department of Energy under the contract number DEFG02-97ER41022. 
[1] R. Bonciani, S. Catani, and P. Mangano, Michelangelo L. and Nason, Nucl. Phys. B529, 424 (1998), hep-ph/9801375.

[2] H. Dreiner and R. J. N. Phillips, Nucl. Phys. B367, 591 (1991).

[3] K. Agashe and M. Graesser, Phys. Rev. D54, 4445 (1996), hep-ph/9510439.

[4] A. Bartl et al., Phys. Lett. B384, 151 (1996), hep-ph/9606256.

[5] F. de Campos et al. (1998), hep-ph/9903245.

[6] S. Bar-Shalom, G. Eilam, and A. Soni, Phys. Rev. D60, 035007 (1999), hep-ph/9812518.

[7] L. Navarro, W. Porod, and J. W. F. Valle, Phys. Lett. B459, 615 (1999), hep-ph/9903474.

[8] T. Han and M. B. Magro, Phys. Lett. B476, 79 (2000), hep-ph/9911442.

[9] A. Belyaev, J. R. Ellis, and S. Lola, Phys. Lett. B484, 79 (2000), hep-ph/0002220.

[10] S. Weinberg, Phys. Rev. D26, 287 (1982).

[11] N. Sakai and T. Yanagida, Nucl. Phys. B197, 533 (1982).

[12] S. Dimopoulos, S. Raby, and F. Wilczek, Phys. Lett. B112, 133 (1982).

[13] L. E. Ibanez and G. G. Ross, Nucl. Phys. B368, 3 (1992).

[14] S. Lola and G. G. Ross, Phys. Lett. B314, 336 (1993).

[15] J. R. Ellis, S. Lola, and G. G. Ross, Nucl. Phys. B526, 115 (1998), hep-ph/9803308.

[16] M. A. Diaz, J. C. Romao, and J. W. F. Valle, Nucl. Phys. B524, 23 (1998), hep-ph/9706315.

[17] A. Pukhov et al. (1999), hep-ph/9908288.

[18] A. Belyaev and A. Gladyshev, to be published.

[19] H. L. Lai et al. (CTEQ), Eur. Phys. J. C12, 375 (2000), hep-ph/9903282.

[20] M. Misiak, S. Pokorski, and J. Rosiek, Adv. Ser. Direct. High Energy Phys. 15, 795 (1998), hep$\mathrm{ph} / 9703442$.

[21] J. L. Diaz-Cruz, H.-J. He, and C. P. Yuan, Phys. Lett. B530, 179 (2002), hep-ph/0103178.

[22] T. Sjostrand, L. Lohnblad, and S. Mrenna, Pythia 6.2: Physics and manual (2001), hep$\mathrm{ph} / 0108264$.

[23] E. D.Richter-Was and L.Poggioli, Atlas note (1998), ATLAS-PHYS-98-131.

[24] W. W. Armstrong et al., Atlas: Technical proposal for a general-purpose $p$ p experiment at the large hadron collider at cern (1994), CERN-LHCC-94-43. 
